\title{
Evaluating the effect of chitosan layer on bioaccessibility and cellular uptake of curcumin nanoemulsions
}

\author{
Hélder D. Silva ${ }^{\mathrm{a}}$, Eliška Beldíkováb ${ }^{\mathrm{b}}$ Joana Poejo ${ }^{\mathrm{c}}$, Luís Abrunhosa ${ }^{\mathrm{a}}$, Ana Teresa Serra ${ }^{\mathrm{d}, \mathrm{e}}$, \\ Catarina M.M. Duarte ${ }^{\mathrm{d}, \mathrm{e}}$, Tomáš Brányik ${ }^{\mathrm{b}}$, Miguel A. Cerqueira ${ }^{\mathrm{f}}$, Ana C. Pinheiro ${ }^{\mathrm{a}, \mathrm{d}, *}$, \\ António A. Vicente ${ }^{\mathrm{a}}$ \\ ${ }^{\text {a }}$ Centre of Biological Engineering, University of Minho, Braga, Portugal \\ b Department of Biotechnology, University of Chemistry and Technology Prague, Technická 5, 166 28, Prague, Czech Republic \\ ${ }^{\mathrm{c}}$ Department of Biochemistry and Molecular Biology, Faculty of Sciences, Institute of Molecular Pathology Biomarkers, University of Extremadura, Badajoz, Spain \\ d IBET, Instituto de Biologia Experimental e Tecnológica, Avenida da República, Quinta-do-Marquês, Estação Agronómica Nacional, Apartado 12, 2781-901, Oeiras, \\ Portugal \\ e Instituto de Tecnologia Química e Biológica António Xavier, Universidade Nova de Lisboa, Av. Da República, 2780-157, Oeiras, Portugal \\ ${ }^{\mathrm{f}}$ International Iberian Nanotechnology Laboratory, Av. Mestre José Veiga s/n, 4715-330, Braga, Portugal
}

\section{A R T I C L E I N F O}

\section{Keywords:}

Nanoemulsions

Lipolysis

Bioaccessibility

Caco- 2 cells

Cellular antioxidant activity

Cellular uptake

\begin{abstract}
A B S T R A C T
Curcumin nanoemulsions stabilized by whey protein isolate were successfully developed using high-pressure homogenization. The effect of a chitosan layer deposition using the layer-by-layer technique on nanoemulsions' stability was evaluated during storage conditions, as well as during gastrointestinal tract passage. Lipids' hydrolysis and curcumin bioaccessibility was assessed using a dynamic gastrointestinal model (simulating the stomach, duodenum, jejunum and ileum) and the cytotoxicity, cellular antioxidant activity and permeability analyses were carried out using Caco- 2 cells. Results showed that both nanosystems were stable during one month of storage and at stomach $\mathrm{pH}$ conditions, whereas creaming and phase separation occurred at intestine $\mathrm{pH}$ conditions. The addition of a chitosan layer increased curcumin bioaccessibility, whereas cellular antioxidant activity studies revealed that nanoemulsions and multilayer nanoemulsions exhibited 9 and 10 times higher antioxidant capacity at the cellular level, respectively, when compared to free curcumin. Permeability assays showed that the use of a chitosan layer significantly increased the apparent permeability coefficient of curcumin through Caco- 2 cells by 1.55 -folds.
\end{abstract}

\section{Introduction}

Curcumin is a natural polyphenolic compound extracted from the rhizome of Curcuma longa, being commercially available as turmeric extract, curcuminoids containing $70-80 \%$ of curcumin, $15-25 \%$ of demethoxycurcumin and $2.5-6.5 \%$ of bisdemethoxycurcumin (Jayaprakasha et al., 2006; Siviero et al., 2015). Curcumin is a food additive (E100) well known for its wide range of beneficial activities, including antioxidant, anti-inflamatory, antimicrobial, anticancer, antiviral, anti-mutagen and wound healing (Jayaprakasha et al., 2006; Kaur et al., 2015; Liu et al., 2016; Siviero et al., 2015). Also, it can act as natural dye in food products and recent studies have shown its capacity to detect heavy metals, which can be of great importance in the field of water and food monitoring (Raj and Shankaran, 2016). However, the use of curcumin is limited by its poor solubility in aqueous media
(11 ng mL ${ }^{-1}$ ), sensitivety to oxygen, $\mathrm{pH}$, solvents and light (PlazaOliver et al., 2015; Siviero et al., 2015; Zhao et al., 2012). Hence, exploitation of curcumin as part of functional foods deeply relies on strategies that overcome these limitations, triggering their incorporation into foods and beverages (Cerqueira et al., 2014; Pinheiro et al., 2016; Plaza-Oliver et al., 2015). Recently, many researchs have sought to overcome these limitations using lipid-based nanosystems (e.g. nanoemulsions composed of only bio-based compounds) to encapsulate liphophilic bioactive compounds, such as curcumin. Nanoemulsions can be used to i) increase their solubility in aqueous media; ii) provide protection from environmental stresses, chemical, enzimatic and oxidative degradation; iii) allow their controlled release; and iv) promote greater bioaccessibility and bioavailability, while also enhancing their permeability of across mucus layer and epithelium cells (Sun et al., 2015; Ting et al., 2014; Zou et al., 2015). Also, the deposition of

\footnotetext{
* Corresponding author. Centre of Biological Engineering, University of Minho, Braga, Portugal.

E-mail addresses: anapinheiro@deb.uminho.pt, anacristinapinheiro@gmail.com (A.C. Pinheiro).
} 
charged polyelectrolyte layers onto oppositelly charged nanoemulsions using the layer-by-layer (LbL) technique, forming multilayer nanoemulsions, can be used as a strategy to further increase the nanoemulsions' physical stability to environmental conditions, control the lipids' digestibility and the release of bioactive compounds in response to specific environmental triggers, while further increasing the bioactive compounds' bioavailability (Acevedo-Fani et al., 2017). Chitosan, a naturally occurring cationic polysaccharide, is one of the polyelectrolytes most used for LbL deposition. It is known to act as a permeation enhancer by opening the tight junctions of the epithelium, facilitating both bioactive compounds' paracellular and transcellular transport. In fact, the positively charged chitosan interacts with the negatively charged mucus to form a complex by ionic or hydrogen bonding, as well as though hydrophobic interactions (Mohammed et al., 2017). The development of tailored nanosystems using the LbL technique relies on their final application, from nanosystems designed to delay lipids' digestability, inducing satiation, to nanosystems designed to enhance permeability across the intestinal membrane, improving bioavailability and consequently, potentiating curcumin's benefitial effects (Maljaars et al., 2009; Pinheiro et al., 2016; Ting et al., 2014).

Despite of the enourmous potential of lipid-based nanosystems, there is still a lack of knowledge about the behavior of these nanosystems after ingestion, from their behavior during digestion, to their absorption through the intestine, bioactive compounds' bioavailability and potential toxicity (McClements, 2013; Pinheiro et al., 2016). Caco2 cell line, derived from human colon adenocarcinoma, is one of the most used intestinal absorption models for studying bioactive compounds' permeability and transport characteristics, once it resembles morphologically the enterocytes of the small intestine (i.e. their monolayers have intercellular tight junctions, microvilli and exhibit brush-border characteristics at the apical side after confluence) (Zeng et al., 2017). Generally, there are two pathways to transport bioactive compounds through the small intestine epithelium which can be modulated to enhance the absorption of bioactive compounds in the gastrointestinal tract: the paracellular and the transcellular pathways (Li et al., 2015a, 2015b; Wang et al., 2015; Yu and Huang, 2012). In paracellular transport, lipid-based nanosystems below $50 \mathrm{~nm}$ may be able to directly diffuse (passive diffusion) through the cell tight junctions (Li et al., 2015b; Wang et al., 2015). Transcellular transport represents the classic digestion-diffusion route, where the bioactive compounds entrapped within lipid-based nanosystems below $500 \mathrm{~nm}$ may be absorbed by the epithelial cells passing through the Caco- 2 cell monolayer via passive or active transport mechanisms (Li et al., 2015b; Yu and Huang, 2012).

The main purposes of this study were the understandment of the behavior of curcumin nanoemulsions and multilayer nanoemulsions under in vitro digestion (e.g. in terms of curcumim bioaccessibility) and the evaluation of their cytotoxicity, cellular antioxidant activity, apparent permeability coefficient and cellular uptake using Caco-2 cell line.

\section{Materials and methods}

\subsection{Materials}

Neobee 1053 medium chain triglycerides (MCTs), a caprylic/capric triglyceride oil with a fatty acid distribution of 55\% of C8:0 and $44 \%$ of C10:0, was kindly provided by Stepan (The Netherlands) and was used without further purification. Curcumin $(\mathrm{Mw}=368.38 \mathrm{Da})$, 1,1-diphenyl-2-picrylhydrazyl (DPPH), the salts used for preparing the gastric and small intestinal electrolyte solutions, hydrochloric acid, sodium bicarbonate, Nile Red 9-diethylamino-5H-benzo[ $\alpha]$ phenoxazine-5-one and dimethyl sulfoxide were purchased from Sigma-Aldrich (St Louis, MO, USA). Whey Protein Isolate (WPI) (Lacprodan DI-9212) was purchased from Arla (Denmark); its protein content was $91 \%$ dry basis, the moisture was $5.5 \%$ in maximum, the ash content was $3 \%$ and the ion content was: sodium, $<0.1 \%$, phosphorus, $0.2 \%$, chloride, $2.2 \%$, potassium, $<0.1 \%$ and calcium, $<0.1 \%$. Chitosan (deacetylation degree $\geq 95 \%$ ) was obtained from Golden-Shell Biochemical CO., LTD (Zhejiang, China) and lactic acid (90\%) from Acros Organics (Geel, Belgium). Sodium hydroxide and phenolphthalein were obtained from Panreac (Spain), chloroform from Fisher Scientific (NJ, USA) and acetone from Fisher Chemical (UK). The reagents for cell-based assays, RPMI 1640 medium, Fetal Bovine Serum (FBS), Penicillin-Streptomycin (PS), trypsin/EDTA and Hanks' Balanced Salt Solution (HBSS) were purchased from Invitrogen (Paisley, UK). Phosphate buffered saline (PBS) powder, 2',7'-dichlorofluorescin diacetate (DCFH-DA), 2,2'Azobis(2-methylpropionamidine) dihydrochloride (AAPH), quercetin and CelLytic ${ }^{\mathrm{TM}}$ MT Cell Lysis Reagent were obtained from Sigma-Aldrich (St. Louis, MO, USA). CellTiter $96^{\circ}$ AQueous One Solution Cell Proliferation Assay (MTS) was obtained from Promega (Wisconsin, USA), Protease Inhibitor Cocktail Set III from Merck Millipore (Darmstadt, Germany), dimethyl sulfoxide (DMSO) from Carlo Erba Reagents Srl (Milan, Italy) and ethanol from Scharlab S.L. (Barcelona, Spain). Distilled water (Milli-Q apparatus, Millipore Corp., Bedford, MA, USA) was used to prepare all solutions.

All the enzymes (i.e. pepsin from porcine gastric mucosa $(600$ $\left.\mathrm{U} . \mathrm{mL}^{-1}\right)$, lipase from porcine pancreas $\left(40 \mathrm{U} \cdot \mathrm{mL}^{-1}\right)$, pancreatin from porcine pancreas $(8 \times$ USP) $)$ and bile extract porcine were purchased from Sigma-Aldrich (St Louis, MO, USA). Caco-2 cells were purchased from Deutsche Sammlung von Mikroorganismen und Zellkulturen (DSMZ, Braunschweig, Germany).

\subsection{Experimental procedures}

\subsubsection{Preparation of curcumin nanosystems}

2.2.1.1. Curcumin nanoemulsions preparation. Curcumin oil-in-water nanoemulsions were prepared according to a previous work (Silva et al., 2015), with minor modifications. Briefly, $0.1 \%(w / w)$ of curcumin was solubilized at $70^{\circ} \mathrm{C}$ in medium chain triglycerides (MCTs) and an aqueous phase containing $1.5 \%(\mathrm{w} / \mathrm{w})$ of whey protein isolate (WPI) was prepared in distilled water. $10 \%$ of MCT solution containing curcumin were homogenized with $90 \%$ of WPI aqueous solution (surfactant/oil ratio (SOR) of 0.135) using an UltraTurrax homogenizer ( $\mathrm{T}$ 25, Ika-Werke, Germany) during $2 \mathrm{~min}$ at $5000 \mathrm{rpm}$, followed by a passage through a high-pressure homogenizer (EmulsiFlex-C3, Avestin, Canada) at 40 bars, during 20 cycles of homogenization (conditions resulting from preliminary tests conducted to optimize processing conditions).

2.2.1.2. Curcumin multilayer nanoemulsions preparation. Multilayer nanoemulsions were formed through the deposition of one chitosan layer onto curcumin nanoemulsions using the LbL electrostatic deposition technique. The saturation method was applied, i.e. the layers were constructed by subsequent adsorption of polyelectrolytes from their solutions without the intermediate rinsing step and avoiding particle aggregation at the same time (Adamczak et al., 2014). Briefly, anionic curcumin nanoemulsions (at $\mathrm{pH} 7$ ) were coated with a layer of positively charged chitosan solution (at $\mathrm{pH} 3$ ), added dropwise with a syringe pump (NE-1000, New Era Pump Systems, Inc., USA) to fresh curcumin nanoemulsions (ratio nanoemulsion:chitosan solution of 1:1), under stirring for $15 \mathrm{~min}$. The $\mathrm{pH}$ values of each solution correspond to the ones at which the solutions present higher charges, in order to maximize the electrostatic interactions between WPI and chitosan. Different concentrations of chitosan were tested in order to optimize the formulation, being the chitosan saturation concentration determined empirically by monitoring the changes in the zeta potential $(Z p)$ and size (Adamczak et al., 2014; Madrigal-Carballo et al., 2010). A screening of chitosan concentration between $0 \%$ and $0.1 \%(\mathrm{w} / \mathrm{w})$ was performed in order to evaluate the influence of chitosan in the nanoemulsions properties, i.e. hydrodynamic diameter $\left(H_{d}\right)$, polydispersity index $(P d I)$ and $Z p$. These properties were measured 
immediately after production.

\subsubsection{Nanosystems size measurements}

Particle size distribution and $P d I$ of nanoemulsions and multilayer nanoemulsions were determined using Dynamic Light Scattering (DLS) (Zetasizer Nano ZS-90, Malvern Instruments, Worcestershire, UK). Nanoemulsions and multilayer nanoemulsions samples were diluted $100 \times$ in distilled water at room temperature. Data was reported as the mean droplet diameter. $P d I$ is a dimensionless and indicates the heterogeneity (monodisperse or polydisperse) of particles size in a mixture (Malvern, 2011). Each sample was analyzed in a disposable polystyrene cell (DTS0012, Malvern Instruments). The measurements were performed in duplicate, with three readings for each of them. The results are given as the average \pm standard deviation of the six values obtained.

\subsubsection{Nanosystems charge measurements}

Droplet charge $(Z p)$ of the nanoemulsions and multilayer nanoemulsions was determined using a particle micro-electrophoresis instrument (Zetasizer Nano ZS-90, Malvern Instruments, Worcestershire, UK). Samples were diluted $100 \times$ in distilled water prior to measurements, in order to avoid multiple scattering effects at ambient temperature, and placed into disposable capillary cells (DTS 1060, Malvern Instruments) (Ozturk et al., 2014). The measurements were performed in duplicate, with three readings for each of them. The results are given as the average \pm standard deviation of the six values obtained.

\subsubsection{Stability of the nanosystems under storage}

In order to evaluate the stability of nanoemulsions and multilayer nanoemulsions during storage, $H_{d}, P d I$ and $Z p$ were evaluated during three months of storage at $4{ }^{\circ} \mathrm{C}$ in the absence of light.

\subsubsection{Nanosystems stability and curcumin release at gastrointestinal environmental conditions}

Curcumin nanosystems stability under gastrointestinal environmental conditions was accessed by a dialysis method. $2 \mathrm{~mL}$ of aqueous curcumin nanosystems were added into a dialysis membrane (molecular weight cut-off $15 \mathrm{kDa}$; Cellu-Sep H1, Membrane filtration products, USA). The sealed dialysis membrane was then placed into $50 \mathrm{~mL}$ of buffer solution (phosphate buffer, PBS, for $\mathrm{pH} 7.4$ and $\mathrm{KCl}-\mathrm{HCl}$ buffer for $\mathrm{pH}$ 2) under magnetic stirring at $37^{\circ} \mathrm{C}$. At appropriate time intervals, $0.5 \mathrm{~mL}$ of supernatant were taken and $0.5 \mathrm{~mL}$ of fresh acceptor medium was added to keep the volume of the release medium constant. Nanosystems stability and the released amount of curcumin from the nanosystems was evaluated by measuring the $H_{d}$ after $54 \mathrm{~h}$ (in order to evaluate if there were changes on nanosystems' behavior over time) and the absorbance at $425 \mathrm{~nm}$ (maximum curcumin's absorbance peak) (Elisa Biotech Synergy HT, Biotek, USA). All stability/release tests were run in triplicate. This assay was only performed to understand the stability of these nanosystems at the $\mathrm{pH}$ and temperature values of the gastrointestinal system.

\subsubsection{In vitro digestion}

2.2.6.1. Dynamic gastrointestinal model. A dynamic gastrointestinal system was used in the in vitro digestion experiments using the methodology developed in a previous work (Pinheiro et al., 2016). This model simulates the main events that occur during digestion and consists of four compartments simulating the stomach, duodenum, jejunum and ileum. Each compartment consists in two connected glass reactors with a flexible wall inside and water is pumped around the flexible walls to maintain the temperature at $37^{\circ} \mathrm{C}$ and to enable the simulation of the peristaltic movements (by the alternate compression and relaxation of the flexible walls). The changes in water pressure are achieved by peristaltic pumps which alter the flow direction according to the time controlled devices connected to them. The compartments are connected by silicone tubes and, at a predefined time, a constant volume of chyme is transferred. All compartments are equipped with $\mathrm{pH}$ electrodes and $\mathrm{pH}$ values are controlled by the secretion of $\mathrm{HCl}$ $\left(1 \mathrm{~mol} . \mathrm{L}^{-1}\right)$ into the stomach and $\mathrm{NaHCO}_{3}\left(1 \mathrm{~mol} . \mathrm{L}^{-1}\right)$ into the intestinal compartments. The gastric and intestinal secretions are added via syringe pumps at pre-set flow rates. The jejunum and ileum compartments are connected with hollow-fiber devices (SpectrumLabs Minikros , M20S-100-01P, USA) to absorb digestion products and water from the chyme and to modify electrolyte and bile salts concentration of the chime (Pinheiro et al., 2016). It should be noted that, although at the mouth stage there may be changes in emulsions' size and interfacial characteristics, influencing the emulsions' behavior in the GI tract (McClements and Xiao, 2012; Pinheiro et al., 2016), this phase was not included once the samples are in the liquid state (and therefore the mastication is not relevant and the residence time in the mouth is very low) and the samples do not contain starch (i.e. the primary enzyme present in saliva, amylase, would not act) (Pinheiro et al., 2016).

2.2.6.2. Experimental conditions. In vitro digestion was performed as described by other authors (Pinheiro et al., 2016) with minor modifications. A volume of $40 \mathrm{~mL}$ of curcumin nanosystems (both nanoemulsions and multilayer nanoemulsions) was introduced into the dynamic gastrointestinal system (gastric compartment) and the experiment was run for a total of $5 \mathrm{~h}$, simulating average physiological conditions of GI tract by the continuous addition of gastric, duodenal, jejunal and ileal secretions. The gastric secretion consisted of pepsin and lipase in a gastric electrolyte solution $(\mathrm{NaCl}$ 4.8 g.L ${ }^{-1}$, KCl 2.2 g.L ${ }^{-1}, \mathrm{CaCl}_{2} 0.22$ g.L $\mathrm{L}^{-1}$ and $\mathrm{NaHCO}_{3} 1.5$ g.L $\mathrm{L}^{-1}$ ), secreted at a flow rate of $0.33 \mathrm{~mL} \mathrm{~min}^{-1}$. The $\mathrm{pH}$ was controlled to follow a predetermined curve (from 4.8 at $\mathrm{t}=0$ to 1.7 at $\mathrm{t}=120 \mathrm{~min}$ ) by secreting $\mathrm{HCl}\left(1 \mathrm{~mol} . \mathrm{L}^{-1}\right)$. The duodenal secretion consisted of a mixture of $4 \%(\mathrm{w} / \mathrm{v})$ porcine bile extract, $7 \%(\mathrm{w} / \mathrm{v})$ pancreatin solution and small intestinal electrolyte solution (SIES) $\left(\mathrm{NaCl} 5 \mathrm{~g} . \mathrm{L}^{-1}, \mathrm{KCl}\right.$ 0.6 g.L $\mathrm{L}^{-1}, \mathrm{CaCl}_{2} 0.25$ g.L $\mathrm{L}^{-1}$ ) secreted at a flow rate of $0.66 \mathrm{~mL} \mathrm{~min}{ }^{-1}$. The jejunal secretion fluid consisted of SIES containing $10 \%(\mathrm{v} / \mathrm{v})$ porcine bile extract solution at a flow rate of $2.13 \mathrm{~mL} \mathrm{~min}^{-1}$. The ileal secretion fluid consisted of SIES at a flow rate of $2.0 \mathrm{~mL} \mathrm{~min}^{-1}$. The $\mathrm{pH}$ in the different compartments of small intestine was controlled by the addition of 1 mol. $\mathrm{L}^{-1} \mathrm{NaHCO}_{3}$ solution to set points of $6.5,6.8$ and 7.2 for simulated duodenum, jejunum and ileum, respectively. During in vitro digestion, samples were collected directly from the lumen of the different compartments and from jejunal/ileal filtrates and ileal delivery. Jejunal and ileal filtrates were used to determine the curcumin's bioaccessibility. The samples were analyzed in terms of $H_{d}$, Zp and free fatty acids (FFA). Both curcumin nanosystems were tested in the dynamic gastrointestinal model at least in triplicate.

\subsubsection{Morphological characterization}

Nanosystems' morphology was evaluated by transmission electron microscopy (TEM) (EM 902A, ZEISS, Germany) operating at $80 \mathrm{kV}$. TEM samples were prepared by depositing the nanoemulsion suspensions on a carbon-coated copper grid, which were negatively stained with $1 \%(\mathrm{w} / \mathrm{v})$ uranyl acetate for observation. Samples were air-dried before analyses. Also, the oil droplets in the emulsions were studied using an epifluorescence microscope (BX51 OLYMPUS, Tokyo, Japan) with an $\times 100$ oil immersion objective lens. Samples were stained with Nile Red (9-diethylamino-5H-benzo[ $\alpha]$ phenoxazine-5-one, $0.25 \mathrm{mg} \mathrm{mL}^{-1}$ in dimethyl sulfoxide, 1:10 (dye:sample), v/v), which enabled the oil droplets to become visible. Slides were prepared by taking $10 \mu \mathrm{L}$ of the stained emulsion solution, placing it in a glass microscope slide and covering with a glass cover slip.

\subsubsection{Free fatty acids release}

The digestion activity was measured by determining the amount of FFA released from curcumin nanoemulsions using a titration method (Pinsirodom and P, 2005). Briefly, $5 \mathrm{~mL}$ of jejunal filtrate, ileal filtrate and ileal delivery samples were collected, $10 \mathrm{~mL}$ of acetone were added 
to quench the enzymes' activity and 3 drops of $1 \%(\mathrm{w} / \mathrm{v})$ phenolphthalein were added as an indicator. A direct titration with 0.1 mol. $\mathrm{L}^{-1} \mathrm{NaOH}$ using a burette was performed and the volume of $\mathrm{NaOH}$ added until the titration end point was determined and used to calculate the concentration of FFA produced by lipolysis. Therefore, the percentage of FFA released was calculated from the number of moles of $\mathrm{NaOH}$ required to neutralize the FFA divided by the number of moles of FFA that could be produced from triglycerides if they were all digested (assuming 2 FFA produced per 1 triacylglycerol molecule) (Li et al., 2011; Pinheiro et al., 2016):

$\% F F A=100 \times\left(\frac{v_{\mathrm{NaOH}} \times m_{\mathrm{NaOH}} \times M_{\text {lipid }}}{w_{\text {lipid }} \times 2}\right)$

where $v_{\mathrm{NaOH}}$ is the volume of sodium hydroxide required to neutralize the FFA generated (in $\mathrm{mL}$ ), $m_{\mathrm{NaOH}}$ is the molarity of the sodium hydroxide used (in mol. $\mathrm{L}^{-1}$ ), $w_{\text {lipids }}$ is the total weight of MCT's oil initially present and $M_{\text {lipid }}$ is the molecular weight of the MCT's oil (based on their average fatty acid composition the molecular weight of MCT's oil was considered to be $503 \mathrm{~g} \mathrm{~mol}^{-1}$ ).

\subsubsection{Curcumin bioaccessibility}

It was assumed that the fraction of the curcumin present in the initial nanosystem that ended up in the micelle phase was a measure of curcumin bioaccessibility (Ahmed et al., 2012) and that the mixed micelles that contained the bioaccessible curcumin fraction were able to pass the hollow-fiber membranes (i.e. corresponds to jejunal filtrate and ileal filtrate samples), while undigested emulsions were retained, according to other authors (Minekus et al., 2005). Curcumin bioaccessibility was determined based on the methodology described by other authors (Ahmed et al., 2012; Pinheiro et al., 2016). Briefly, $5 \mathrm{~mL}$ of the sample (jejunal or ileal filtrate) were vortexed with $5 \mathrm{~mL}$ of chloroform and then centrifuged (Sigma 4K15, Germany) at $1750 \mathrm{rpm}$, at room temperature, for $10 \mathrm{~min}$. The bottom chloroform layer was collected and the extraction procedure was repeated with the top layer. The second bottom chloroform layer was added to the previously set aside chloroform layer, mixed, and analyzed in a UV-VIS spectrophotometer (Elisa Biotech Synergy HT, Biotek, USA) at $425 \mathrm{~nm}$ (absorbance peak). The concentration of curcumin was determined from a previously prepared calibration curve of absorbance versus curcumin concentration in chloroform.

\subsubsection{Cell based assays}

2.2.10.1. Cell culture. Caco-2 cell line was selected for cytotoxicity experiments, cellular antioxidant activity evaluation and permeation studies. Caco- 2 cell line were routinely grown in RPMI 1640 culture medium supplemented with $10 \%(\mathrm{v} / \mathrm{v})$ of inactivated FBS and $1 \%(\mathrm{v} / \mathrm{v})$ of PS. Stock cells were maintained as monolayers in $75 \mathrm{~cm}^{2}$ culture flasks. Cells were subcultured every week at a split ratio of 1-4 by treatment with trypsin/EDTA $(0.25 \%)$ and incubated at $37^{\circ} \mathrm{C}$ in a $5 \%$ $\mathrm{CO}_{2}$ humidified atmosphere (Matias et al., 2014). For all cell-based assays Caco- 2 were used between passages 30 and 50 .

2.2.10.2. Cytotoxicity assay. Caco-2 cells were seeded at a density of $2 \times 10^{4}$ cells/well in 96-well plate and the medium was changed every $48 \mathrm{~h}$. The experiments were performed through method previously described by other authors, using completely differentiated cells (after reaching confluence, $\sim 96 \mathrm{~h}$ ) (Serra et al., 2011b, 2013). An initial stock solution of curcumin in ethanol was prepared $\left(4 \mathrm{mg} \mathrm{mL}^{-1}\right)$. In the day of the assay, curcumin, nanoemulsions and multilayer nanoemulsions were diluted in RPMI medium supplemented with $0.5 \%$ inactivated FBS $\left(4.8-19.0 \mu \mathrm{g}_{\text {curcumin }} \cdot \mathrm{mL}^{-1}\right.$ and $31.25-250.00$ $\mu g_{\text {chitosan }} \cdot \mathrm{mL}^{-1}$ ) and added to Caco-2 cells in triplicate. After $4 \mathrm{~h}$ of incubation at $37^{\circ} \mathrm{C}$ in a $5 \% \mathrm{CO}_{2}$ humidified atmosphere, samples were removed and cells were washed twice with PBS. Then, $100 \mu \mathrm{L}$ of CellTiter $96^{\circ}$ AQueous One Solution Cell Proliferation Assay reagent
(MTS) diluted in RPMI 1640 medium supplemented with $0.5 \%$ FBS was added to each well and left to react for $2 \mathrm{~h}$, at $37^{\circ} \mathrm{C}$ in a $5 \% \mathrm{CO}_{2}$ humidified atmosphere. MTS enters in cells and it is bio-reduced into a colored formazan product that is soluble in the culture medium. The quantity of formazan produced was measured spectrophotometrically at $490 \mathrm{~nm}$ in a microplate reader (EPOCH, Bio-Tek, USA) and is directly proportional to the number of living cells in culture. Results were expressed in terms of percentage of cellular viability relative to a group control (cells only with RPMI medium). Experiments were performed in triplicate in three independent assays.

2.2.10.3. Cellular antioxidant activity. Cellular Antioxidant Activity (CAA) was determined using the method previously described by other authors with some modifications (Serra et al., 2011a). In this assay, dichlorofluorescein is trapped in the cells and is oxidized to fluorescent dichloroffluorescein. This method measures the ability of antioxidants to prevent the formation of fluorescent dichloroffluorescein by AAPH-generated peroxyl radicals(Serra, 2010; Wang and Joseph, 1999). Thus, the decrease in the cellular fluorescence when compared to the control cells indicates the antioxidant activity of curcumin. Briefly, Caco- 2 cells were seeded at a density of $2 \times 10^{4}$ cells/well in a 96-well plate and the medium was changed every $48 \mathrm{~h}$. After reaching confluence $(\sim 96 \mathrm{~h})$, cells were washed twice with PBS and triplicate wells were treated for $1 \mathrm{~h}$ with $100 \mu \mathrm{L}$ of different concentrations of curcumin $\left(5-40 \mu \mathrm{g} \mathrm{mL}^{-1}\right)$ or nanoemulsions and multilayer nanoemulsions $\left(0.95-7.60 \mu \mathrm{gL}^{-1}\right)$ plus $25 \mu \mathrm{mol} . \mathrm{L}^{-1}$ of DCFH-DA diluted in PBS. Then, the medium was removed, cells were washed with PBS and $100 \mu \mathrm{L}$ of $600 \mu \mathrm{mol} \mathrm{L}^{-1}$ of AAPH was added in each well. The 96 -well microplate was placed into a fluorescence reader (FL800, Bio-Tek Instruments, Winooski, VT, USA) at $37^{\circ} \mathrm{C}$. Emission at $530 \pm 25 \mathrm{~nm}$ was measured after excitation at $485 \pm 20 \mathrm{~nm}$ every $5 \mathrm{~min}$ for $1 \mathrm{~h}$. Each plate included triplicate control and blank wells: control wells contained cells treated with DCFH-DA and oxidant (AAPH); blank wells contained cells treated with DCFH-DA without oxidant. Other control test has been performed, which consisted in wells containing cells treated with DCFH-DA with just water or ethanol, in order to evaluate the ethanol (used to dilute free curcumin) oxidation capacity. Quercetin, previously dissolved in DMSO $\left(20 \mathrm{mmol} . \mathrm{L}^{-1}\right)$ was used as standard $\left(1.25-10.00 \mu \mathrm{mol} . \mathrm{L}^{-1}\right)$ for the calibration curve. CAA of samples was quantified as described by other authors (Wolfe and Liu, 2007). Briefly, after blank and initial fluorescence subtraction, the area under the curve for fluorescence versus time was integrated to calculate the CAA value at each concentration of the sample:

$C A A$ unit $=1-\left(\int \frac{S A}{C A}\right)$

Where $\int S A$ is the integrated area under the sample fluorescence versus time curve and $\int C A$ is the integrated area of the control curve. The median effective concentration $\left(E C_{50}\right)$ was determined for the sample from the median effect plot of $\log \left(f_{a} / f_{u}\right)$ versus $\log$ (dose), where $f_{\mathrm{a}}$ is the fraction affected (CAA unit) and $f_{\mathrm{u}}$ is the fraction unaffected (1CAA unit) by the treatment. The $E C_{50}$ values were stated as mean $\pm \mathrm{SD}$ for triplicate sets of data obtained from the same experiment. Also, $E C_{50}$ were converted to $C A A$ values, expressed as micromoles of quercetin per mg of curcumin, using the mean $E C_{50}$ value of quercetin from three independent experiments, $3.52 \pm 0.42 \mu \mathrm{mol}^{-\mathrm{L}^{-1}}$.

2.2.10.4. Permeability studies. For transport studies, Caco-2 cells were seeded in $12 \mathrm{~mm}$ i.d. Transwell ${ }^{\circ}$ inserts (polycarbonate membrane, $0.4 \mu \mathrm{m}$ pore size, Corning Costar Corp.) in 12-well plates at a density of $1 \times 10^{5}$ cells/well. The basolateral (serosal) and apical (mucosal) compartments contained 1.5 and $0.5 \mathrm{~mL}$ of culture medium, respectively. Cells were allowed to grow and differentiate to confluent monolayers for 21 days by changing the medium (RPMI 1640 supplemented with $10 \%$ of FBS and $1 \%$ of PS) three times a week (Serra, 2010). By culturing them for 21 days, Caco-2 cells are 
spontaneously differentiated and tight junctions are formed between the cells, microvillus structures are formed on the apical cell surface and a variety of brush-border digestive enzymes, transporters and receptors are expressed (Langerholc et al., 2011; Shimizu, 2010). Transepithelial electrical resistance (TEER) of cells grown in Transwell was measured using an epithelial Volt-Ohm-meter (WPI, Berlin, Germany). The measurement of electrical resistance across the monolayer is a measure of passive ion transport across the cell and cell junctions and can be used as a criterion for the tightness of the cell monolayer in order to evaluate and determine the monolayer integrity. The TEER value was measured from the following equation:

TEER $=\left(R_{\text {monolayer }}-R_{\text {blank }}\right) \times A$

where $R_{\text {monolayer }}$ is the resistance of the cell monolayer along with the filter membrane, $R_{\text {blank }}$ is the resistance of the filter membrane and $A$ is the surface area of the membrane $\left(1.12 \mathrm{~cm}^{2}\right.$ in 12 -well plates). Only monolayers with TEER value higher than $500 \Omega \mathrm{cm}^{2}$ were used for experiments (Matias et al., 2014).

In the day of the experiment, the medium was removed and cells were washed twice with $\mathrm{HBSS}\left(\mathrm{pH} 7.4,37^{\circ} \mathrm{C}\right)$ to remove traces of RPMI medium. After washing, cells were incubated with transport buffer (HBSS) for $60 \mathrm{~min}$ at $37^{\circ} \mathrm{C}$ in a $5 \% \mathrm{CO}_{2}$ incubator. For permeability transport studies, $500 \mu \mathrm{L}$ of nanosystems (nanoemulsions and multilayer nanoemulsions) diluted in HBSS $\left(19 \mu \mathrm{g}_{\text {curcumin }} \cdot \mathrm{mL}^{-1}\right)$ was added to the apical compartment and $1.5 \mathrm{~mL}$ of transport buffer to the basolateral side. The transepithelial transport was followed as a function of time: after $0,15,30,45,60$ and $120 \mathrm{~min}$ of incubation, TEER was measured and the basolateral and apical samples were collected and frozen until analysis. The apparent permeability coefficient $\left(P_{\text {app }}\right)$ of nanosystems were calculated using the following equation:

$P_{a p p}=\frac{\frac{d Q}{d t}}{\operatorname{Co} \times A}$

where $d Q / d t$ is the cumulative transport rate $\left(\mu \mathrm{g} \cdot \mathrm{min}^{-1}\right)$ defined as the slope obtained by linear regression of cumulative transported amount as a function of time ( $\mathrm{min}$ ), Co is the initial nanosystems concentration in the donor compartment and $A$ is the membrane surface area (1.12 $\mathrm{cm}^{2}$ in 12-well plates).

In addition, the cellular uptake of curcumin was also quantified. For this purpose, after 15, 30, 45, 60 and $120 \mathrm{~min}$ of incubation with samples, the Caco- 2 monolayer was washed with $100 \mu \mathrm{L}$ of HBSS to remove traces of nanosystems from the apical compartment and then cells were incubated for 5 min with $100 \mu \mathrm{L}$ of CelLytic ${ }^{\mathrm{TM}}$ MT Cell Lysis Reagent containing 1\% (v/v) of Protease Inhibitor Cocktail (Matias et al., 2014). After scrapping, cells were collected and frozen until quantitative analysis by the high performance liquid chromatography (HPLC) method described below.

\subsubsection{HPLC analysis of curcumin}

HPLC system comprised a Varian Prostar 210 pump, a Varian Prostar 410 autosampler and a Jasco FP-920 fluorescence detector $\left(\lambda_{\mathrm{exc}}=420 \mathrm{~nm}\right.$ and $\left.\lambda_{\mathrm{em}}=540 \mathrm{~nm}\right)$. The instrument and the chromatographic data were managed by a Varian 850-MIB data system interface and a Galaxie chromatography data system, respectively. The chromatographic separation was performed on a C18 reversed-phase YMC-Pack ODS-AQ analytical column $(250 \times 4.6 \mathrm{~mm}$ I.D., $5 \mu \mathrm{m})$ that was fitted with a pre-column with the same stationary phase. The compounds were eluted using a flow rate of $1.0 \mathrm{~mL} \mathrm{~min}^{-1}$ during a $15 \mathrm{~min}$ isocratic run at a temperature of $30^{\circ} \mathrm{C}$. The injection volume was $20 \mu \mathrm{L}$. The mobile phase was a mixture of acetic acid $(2 \% \mathrm{v} / \mathrm{v}), \mathrm{pH}$ 2.5 , and acetonitrile (at a ratio of $47: 53 \mathrm{v} / \mathrm{v}$ ) that was filtered and degassed with a $0.2 \mu \mathrm{m}$ membrane filter (GHP, Gelman). A calibration curve was prepared with standard solutions containing $0.1 \mu \mathrm{g} \mathrm{mL}{ }^{-1}$ to $10.0 \mu \mathrm{g} \mathrm{mL}^{-1}$ of curcumin in acetonitrile. Curcumin, demethoxycurcumin, and bisdemethoxycurcumin were quantified by comparing the peak areas with the calibration curve. Retention times were 10.5, 11.5 , and $12.5 \mathrm{~min}$, respectively.

\subsection{Statistical procedures}

\subsubsection{Data analyses}

Data analyses were performed using Microsoft Windows Excel 2011, using the Tukey's Multiple Comparison Test with a confidence interval of $95 \%$ in GraphPad Prism 5 (GraphPad Software, Inc.) and using ANOVA in STATISTICA 7.0 (Statsoft, Tulsa, OK, USA).

Regarding the cell assays all data are expressed as means \pm standard error and individual experiments were performed at least in triplicate. The statistical analysis was done using SigmaStat $3.0^{\circ}$ software. All values were tested for normal distribution and equal variance. When homogeneous variances were confirmed, data were analyzed by One Way Analysis of Variance (ANOVA) coupled with the Tukey's posthoc analysis to identify means with significant differences ( $p$ value of $p<0.05$ was considered significant).

\section{Results and discussion}

\subsection{Curcumin nanosystems development and characterization}

Different concentrations of WPI were tested aiming at preparing curcumin nanoemulsions using $10 \%(\mathrm{w} / \mathrm{w})$ of MCTs as the oily phase (results not shown). The minimum concentration of WPI required to form stable nanoemulsions was found to be $1.5 \%(\mathrm{w} / \mathrm{w})$ presenting values of $H_{d}$ of $186 \pm 3.9 \mathrm{~nm}$ and a $P d I$ of $0.124 \pm 0.014$, regarding the electrical charge $(Z p)$ a value of $-51.9 \pm 2.4 \mathrm{mV}$ was obtained.

Fig. 1a shows that the addition of chitosan results in a change of $Z p$ values, where the increase of chitosan concentrations $(0 \%-0.1 \%)$ changes the $Z p$ values from highly negative to highly positive values, reaching a positive constant value around $42 \mathrm{mV}$, when the concentration ranged between $0.05 \%$ and $0.1 \%(\mathrm{w} / \mathrm{w})$. This saw-like profile in the $Z p$ values indicates that chitosan was adsorbed to the surface of the nanoemulsions, forming multilayer nanoemulsions (Szczepanowicz et al., 2015). At chitosan concentrations ranging from $0.01 \%$ to $0.02 \%(\mathrm{w} / \mathrm{w})$ large "clumps" and oily droplets were observed at the upper surface of the samples. This behavior can be explained by the insufficient amount of chitosan present to coat all the nanoemulsions droplets, reflected by the $Z p$ of the samples that achieved an increase from -51.9 for the nanoemulsions to only -15.1 and $0.8 \mathrm{mV}$ for $0.01 \%$ and $0.02 \% \mathrm{w} / \mathrm{w}$, respectively. In Fig. $1 \mathrm{~b}$ it is possible to observe the effect of these large clumps in the $H_{d}$ of the samples, increasing the sizes and presenting a high PdI. The presence of clumps indicates that bridging flocculation occurred. Charged polyelectrolytes can trigger bridging flocculation, as there is an insufficient amount of chitosan to completely coat the nanoemulsions, chitosan could link to the surface of more than one nanoemulsion droplet coupling nanoemulsions together (Cui et al., 2014; Guzey and McClements, 2006; Mora-Huertas et al., 2010). Similar behaviors were presented by Aoki et al. (2005) and Mun et al. (2005). Both authors verified that concentrations of chitosan below the saturation point resulted in droplets with negative surfaces, allowing chitosan to adsorb to the surface of two or more droplets simultaneously, forming large aggregates and clumps (Aoki et al., 2005; Mun et al., 2005).

From $0.02 \%$ to $0.1 \%(\mathrm{w} / \mathrm{w})$ a change from neutral to highly positive $Z p$ was observed, suggesting that chitosan adsorbed to the surface of the nanoemulsions (Szczepanowicz et al., 2015). However, the effect of $\mathrm{pH}$ decrease in WPI charge should not be excluded and could also contributed to this high positive $Z p$ value. Increasing the chitosan concentration above $0.05 \% \mathrm{w} / \mathrm{w}$ did not significantly increased $(p>0.05)$ the $Z p$ value, leading to a constant value around $42 \mathrm{mV}$, however resulted in a significant $(p>0.05)$ increase of the $H_{d}$ values from $189.1 \pm 3.4$ to $198.7 \pm 5.4 \mathrm{~nm}$, for $0.05 \%$ and $0.1 \%(\mathrm{w} / \mathrm{w})$ of chitosan, respectively. It also promoted an increase of $P d I$ values from 

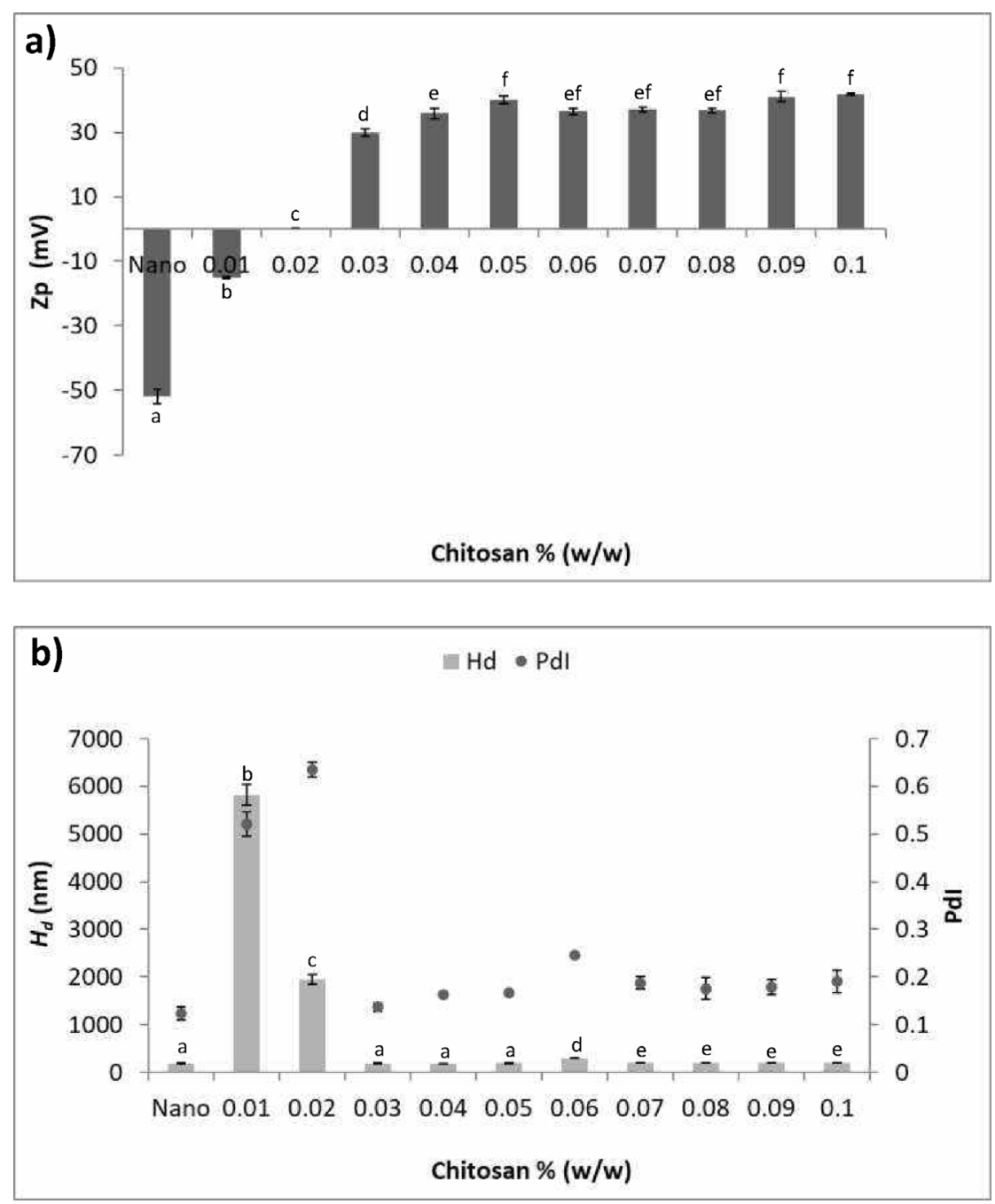

Fig. 1. Development of multilayer nanoemulsions as a function of chitosan concentrations: a) Change in the zeta potential ( $\mathrm{Zp}$ ); b) Change in the hydrodynamic diameter $\left(H_{d}\right)$ and polydispersity index $(P d I)$. Each value represents mean $\pm \mathrm{SD}(\mathrm{n}=6)$; One Way Analysis of Variance (ANOVA) coupled with the Tukey's post-hoc analysis to identify means with significant differences ( $p$ value of $p<0.05$ was considered significant); ${ }^{\text {a-f }}$ Different superscripts indicate significant differences among samples.

$0.167 \pm 0.004$ to $0.191 \pm 0.024$, for the same range of concentrations. Results suggest that the nanoemulsions become saturated with chitosan, where the strong electrostatic repulsions present prevented droplet aggregation by bridging and depletion flocculation (Li et al., 2010; Pinheiro et al., 2016). Hence, $0.05 \%$ (w/w) of chitosan was selected for the build-up of the multilayer nanoemulsions, without significant excess of polyelectrolytes in solution. The developed multilayer nanoemulsions presented a final $H_{d}$ of $189 \pm 3.4 \mathrm{~nm}$, with a $P d I$ of $0.167 \pm 0.004$ and a $Z p$ value of $40.1 \pm 1.2 \mathrm{mV}$. TEM and fluorescence microscopy confirmed the mean droplet diameters achieved and confirmed the development of nanosystems with spherical morphology (Fig. 2, microphotographs of initial nanoemulsions and multilayer nanoemulsions). Also note that nanoemulsions probably have a quite rigid surface coating on the droplets, once they remained stable and spherical after the drying process applied in sample preparation for TEM analysis.

\subsection{Nanosystems stability under storage conditions}

Both developed nanosystems showed no macroscopic sign of instability phenomena (i.e. creaming or phase separation) after 35 days of storage. Fig. 3 shows that curcumin nanoemulsions and multilayer nanoemulsions maintained their $H_{d}$ during storage. These results show that the storage conditions used $\left(4^{\circ} \mathrm{C}\right.$ in the absence of light) did not statistically influence $(p>0.05)$ the $H_{d}$ values when compared to the values obtained after nanosystems production (Fig. 3).

Similar results were obtained by other authors, which showed that curcumin nanoemulsions also stabilized by a bio-based surfactant (in this case, with $2.0 \%$ lecithin) did not suffer destabilization phenomena during almost 86 days of experiment (Artiga-Artigas et al., 2018).

\subsection{Evaluation of nanosystems responsiveness at gastrointestinal environmental conditions}

The stability/responsiveness of both nanosystems was accessed at gastrointestinal $\mathrm{pH}$ and temperature conditions (i.e. at $\mathrm{pH} 2$ and 7.4 and $37^{\circ} \mathrm{C}$ ) during $54 \mathrm{~h}$, by performing release assays and by measuring the absorbance of curcumin in the acceptor mediums. Also, after $54 \mathrm{~h}$ of release assays, the $H_{d}$ and $P d I$ of the nanosystems were evaluated in order to determine the changes in these parameters over time.

At $\mathrm{pH} 7.4$ none of the nanosystems released curcumin during the evaluation period, maintaining curcumin entrapped within the nanosystems. Considering the $H_{d}$ stability, it was observed that after $54 \mathrm{~h}$ of assay, at $\mathrm{pH} 7.4$, the nanosystems maintained values of $H_{d}$ closer $(p>0.05)$ to the initial value, with $187.3 \pm 2.7 \mathrm{~nm}$ for the nanoemulsions and $189.1 \pm 2.8 \mathrm{~nm}$ for the multilayer nanoemulsions. At these conditions, i.e. $\mathrm{pH}$ above the $\mathrm{pK}_{\mathrm{a}}$ of chitosan, the amino groups of 


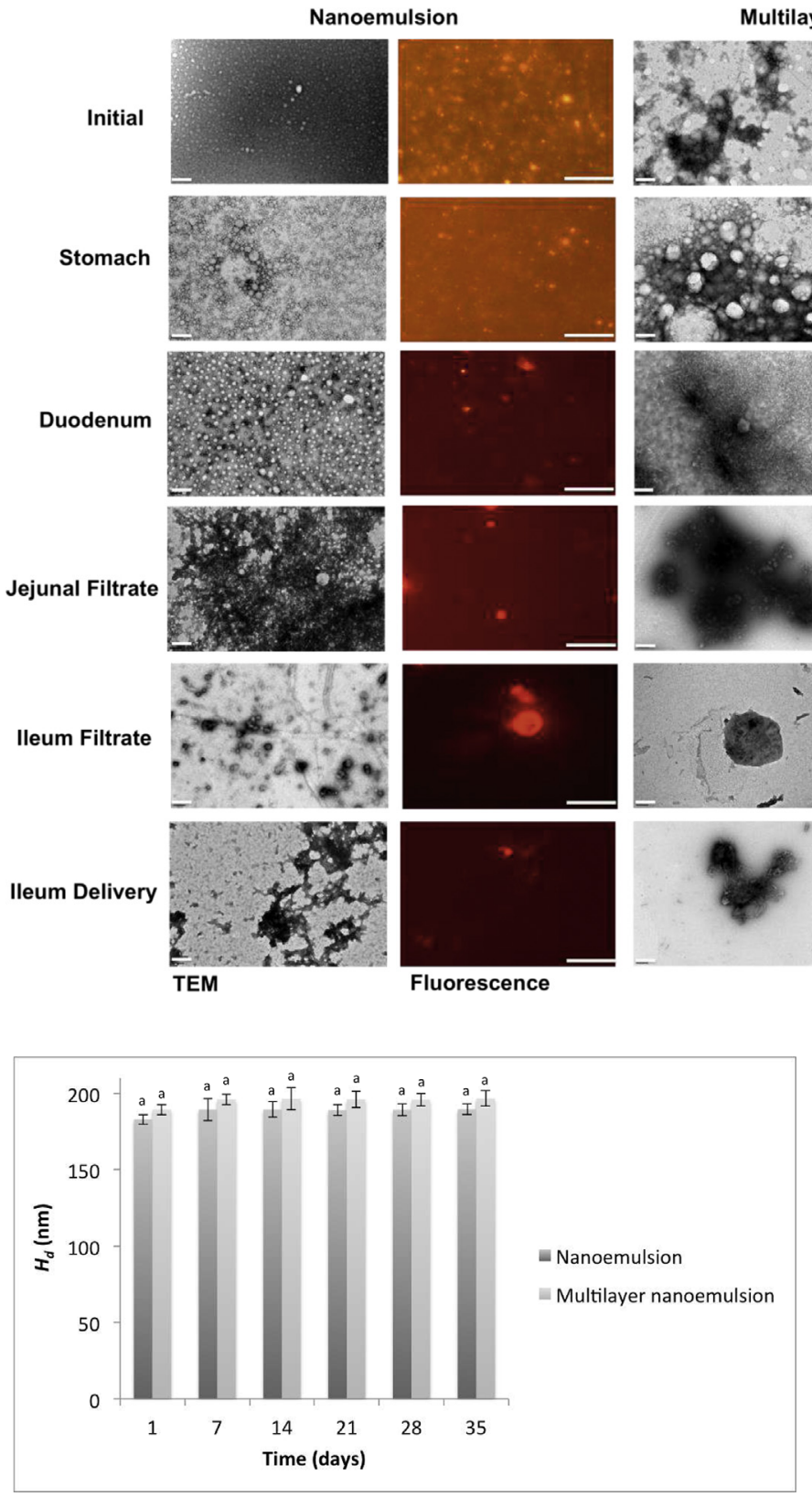

Fig. 3. Hydrodynamic diameter $\left(H_{d}\right)$ during 35 days of storage for nanoemulsion and multilayer nanoemulsion. Each value represents mean $\pm \mathrm{SD}(n=6)$; One Way Analysis of Variance (ANOVA) coupled with the Tukey's post-hoc analysis to identify means with significant differences ( $p$ value of $p<0.05$ was considered significant).

chitosan are entirely deprotonated (Vachoud et al., 2000), although, it is feasible that chitosan held some positive charge. Previous works showed that $\mathrm{pK}_{\mathrm{a}}$ of charged groups can change when entangled between polyelectrolytes of oppositely charges, improving the stability of the multilayer nanoemulsions to $\mathrm{pH}$ changes (Burke and Barrett, 2003a; b; Li et al., 2010).

At $\mathrm{pH}$ 2, the nanoemulsions loss WPI to the acceptor medium (data not shown), being unstable at this $\mathrm{pH}$ after $54 \mathrm{~h}$ of assay, thus rendering it impossible to measure $H_{d}$. However, for the multilayer nanoemulsions, the $H_{d}$ remained stable after $54 \mathrm{~h}$ of assay $\left(H_{d}=190.4 \pm 3.1 \mathrm{~nm}\right)$, while no sign of curcumin was present in the acceptor medium $\left(0 \mu \mathrm{gL}^{-1}\right.$, below the detection limit of $0.01 \mu \mathrm{g} \mathrm{m}^{-1}$ ). Curcumin is reported as having low solubility in aqueous systems, $11 \mathrm{ng} \mathrm{mL}^{-1}$, which could explain the absence of
Fig. 2. TEM microphotographs of negatively stained nanosystems with uracyl $1 \% \quad \mathrm{w} / \mathrm{w}$ and epifluorescence microphotographs stained with Nile Red as they undergo through the dynamic in vitro digestion. The scale bar for all TEM images and epifluorescent images are $400 \mathrm{~nm}$ and $20 \mu \mathrm{m}$, respectively. (For interpretation of the references to color in this figure legend, the reader is referred to the Web version of this article.) curcumin release (Jelezova et al., 2015; Zhao et al., 2012). The fact that at $\mathrm{pH} 2$ the loss of WPI only occurred in nanoemulsions suggests that the chitosan layer was able to protect the WPI-stabilized nanoemulsions, improving their stability at acidic $\mathrm{pH}$.

\subsection{Dynamic in vitro digestion}

LbL technique can be used to modify the interfacial composition of nanoemulsions increasing their stability under gastrointestinal tract conditions, delaying lipid digestion (Hu et al., 2011; Klinkesorn and Julian McClements, 2010; Yang et al., 2014). These experiments evaluated the influence of a chitosan layer in nanoemulsions behavior under in vitro digestions, mimicking the human gastrointestinal tract.

\subsubsection{Influence of chitosan on nanosystems characteristics during in vitro digestion}

The effect of the deposition of a chitosan layer on the nanoemulsions' $H_{d}$, morphology and $Z p$ was evaluated at each stage of the in vitro digestion. Initially the nanosystems had a $H_{d}$ around 186 and $189 \mathrm{~nm}$ for the nanoemulsions and multilayer nanoemulsions, respectively. After being subjected to stomach conditions, the nanoemulsions $H_{d}$ values significantly increased $(p<0.05)$ to $258.3 \pm 26.7 \mathrm{~nm}$ (Fig. 4a) evidencing some coalescence phenomena, confirmed by microscopy images that shows an increase in droplet size (Fig. 2). For multilayer nanoemulsions, the $H_{d}$ value obtained under the gastric conditions was $205.4 \pm 16.8 \mathrm{~nm}$, which does not present a statistically significant difference $(p>0.05)$ when compared with the initial value. From microscopy images (Fig. 2) some larger droplets can be observed, nonetheless droplets' size remained in the nano-size range. This behavior can be related with the interfacial characteristics of the nanosystems and their surface charge. Under gastric conditions ( $\mathrm{pH}$ around 2), both nanosystems are positively charged: nanoemulsions presented a positive $Z p$ of $29.7 \pm 5.3 \mathrm{mV}$ and the multilayer nanoemulsions presented a $Z p$ of $36.6 \pm 3.0 \mathrm{mV}$ (Fig. $4 \mathrm{~b}$ ), exhibiting strong electrostatic 

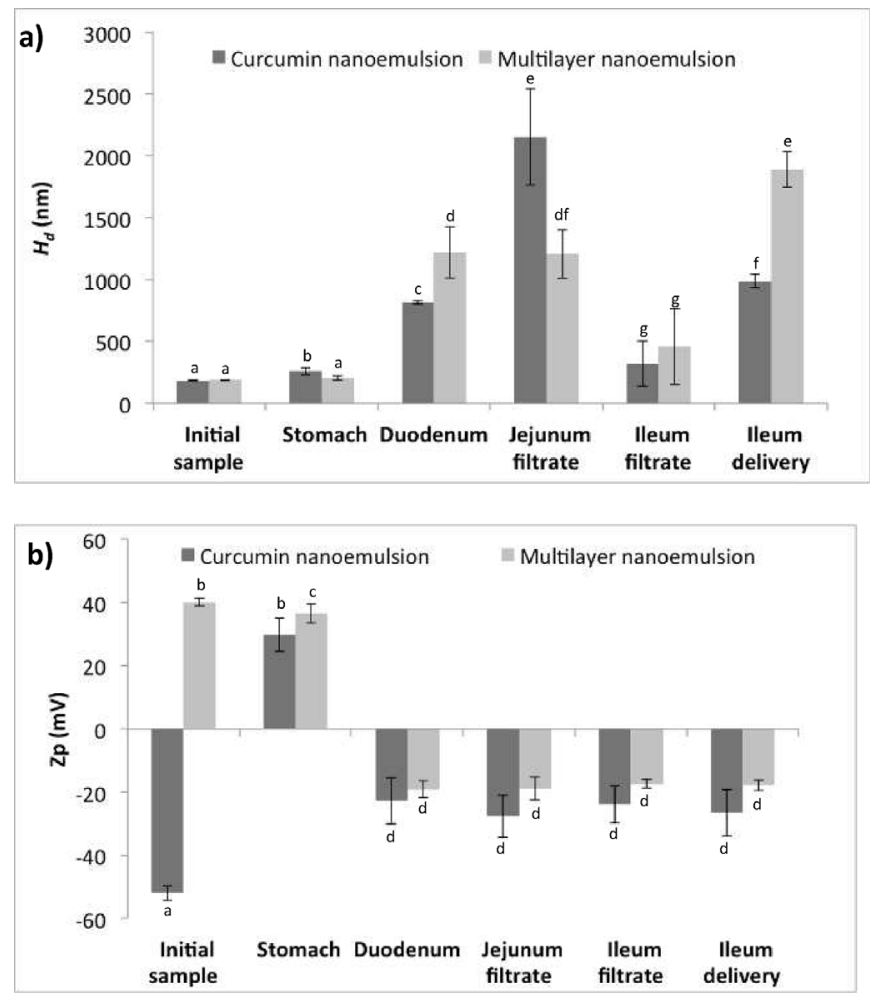

Fig. 4. a) Hydrodynamic diameter $\left(H_{d}\right)$ for the nanosystems as they undergo through the dynamic in vitro digestion; b) Zeta potential values $(Z p)$ for the nanosystems as they undergo through the dynamic in vitro digestion. Each value represents mean $\pm \operatorname{SD}(n=6)$; One Way Analysis of Variance (ANOVA) coupled with the Tukey's post-hoc analysis to identify means with significant differences ( $p$ value of $p<0.05$ was considered significant); ${ }^{\mathrm{a}-\mathrm{g}}$ Different superscripts indicate significant differences among samples.

repulsion between droplets and thus avoiding droplet aggregation, flocculation and coalescence (Klinkesorn and Julian McClements, 2010; Pinheiro et al., 2016; Zhang et al., 2015). The change in the $Z p$ values, from negative to positive, for nanoemulsions at gastric conditions can be attributed to changes in solutions conditions, $\mathrm{pH}$ and ionic strength. In fact, at pHs below WPI isoelectric point ( $\mathrm{pI} \sim 5.2$ ), the WPI-stabilized nanoemulsions would be positively charged, which is in agreement with other published works (Rodrigues et al., 2015).

Under intestinal conditions, both nanosystems exhibited an increase in the $H_{d}$ due to particle aggregation either by flocculation or coalescence of droplets (Figs. 2 and 4). At the duodenal stage, the $H_{d}$ values increased to $815.2 \pm 13.5 \mathrm{~nm}$ for nanoemulsions, presenting a $Z p$ value of $-22.7 \pm 7.2 \mathrm{mV}$; in the case of the multilayer nanoemulsion, the $H_{d}$ increased to $1218.6 \pm 205.7 \mathrm{~nm}$ and the $Z p$ to $-19.1 \pm 2.7 \mathrm{mV}$. Microscopy analyses showed that at this stage, both nanosystems exhibited droplets at the nano and micro-scale, confirming the $H_{d}$ values presented in Fig. 4a. At jejunum and ileum stages both nanosystems presented a high $H_{d}$. For all of the intestinal stages of digestion, sizes at the micro-scale were found (Fig. 2), still, TEM analyses showed some structures at the nano-scale.

The results from the evaluation of nanosystems responsiveness at gastrointestinal pHs (section 3.3) revealed that both nanosystems are more stable at intestinal $\mathrm{pH}$, however, with the addition of all the other gastrointestinal components (e.g. enzymes, bile salts, simulation of peristaltic movements), the higher changes in nanosystems' properties occurred at the small intestinal stages. This suggests that the higher nanosystems' instability (e.g. higher $H_{d}$ ) observed at the these stages are due to the ability of bile salts to displace WPI and chitosan from the droplets' surface and to the capacity of lipase to conduct the hydrolysis of the triacylglycerol molecules present into FFA, monoacyglycerides

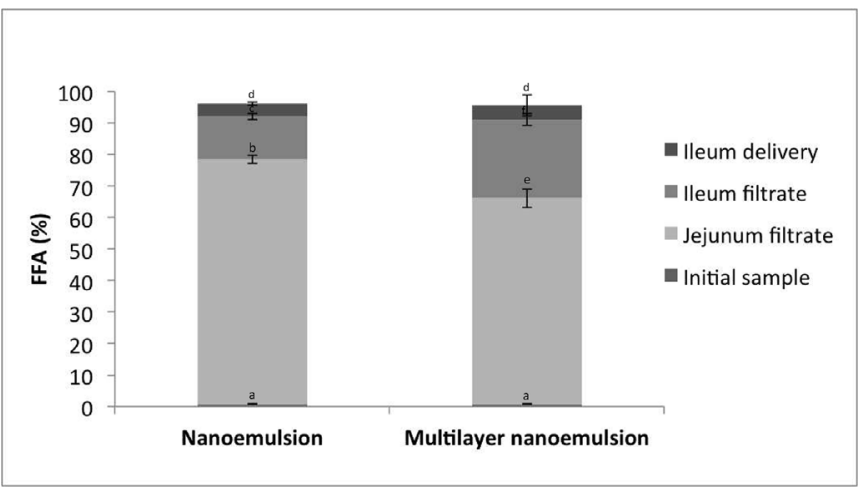

Fig. 5. Percentage of free fatty acids (FFA) released from the nanosystems as they undergo through the dynamic in vitro digestion. Each value represents mean $\pm S D(n=3)$; One Way Analysis of Variance (ANOVA) coupled with the Tukey's post-hoc analysis to identify means with significant differences ( $p$ value of $p<0.05$ was considered significant); ${ }^{\text {a-f }}$ Different superscripts indicate significant differences among samples.

and/or diacylglycerides (Pinheiro et al., 2016). This hydrolysis can also generate structures such as micelles, vesicles or other colloidal structures, which also contributed to the changes observed in $H_{d}$ and morphology of the nanosystems ( $M u$ and Høy, 2004).

Regarding the effect of intestinal conditions on the droplet charge, results show that the $Z p$ values decreased, changing from positive to negative charges for both nanosystems, reaching to values ranged between $-28 \mathrm{mV}$ and $-23 \mathrm{mV}$ for the nanoemulsions and around $-18 \mathrm{mV}$ for the multilayer nanoemulsions (Fig. 4b). These results suggest that the anionic components present in the intestinal juices (e.g. bile salts and FFA) might have displaced the WPI and chitosan from droplet surface or that bile salts or FFA adsorbed onto the surface of the nanosystems, conferring the negative charge (Klinkesorn and Julian McClements, 2010; Pinheiro et al., 2016; Salvia-Trujillo et al., 2015; Zou et al., 2015). There were no significant differences $(p>0.05)$ between the values of $Z p$ of both nanosystems at intestinal conditions.

\subsubsection{Influence of chitosan layer on lipids digestion}

The effect of chitosan layer in the lipids digestion was examined by determining the release of FFA, generated by the hydrolysis of the triacylglycerol molecules, at each stage of the small intestine digestion (Fig. 5). The deposition of a chitosan layer onto nanoemulsions did not had a significant effect $(p>0.05)$ on the extent of lipid digestion, achieving a similar overall extent of released FFA (Fig. 5) of $96.14 \pm 1.36 \%$ and $95.52 \pm 4.93 \%$ for nanoemulsions and multilayer nanoemulsions, respectively. These results suggest that the chitosan layer did not prevent lipid digestion, rather delayed it during the jejunum stage, which might be indicated its degradation or disintegration. In fact, some studies suggest that pancreatic lipase has the ability to hydrolyze chitosan, explaining the lack of efficiency of the developed layer (Pantaleone et al., 1992; Shin et al., 2001). Nonetheless, this study shows some differences in the rate at which the nanosystems were digested. In Fig. 5 it is possible to see that in the jejunum, the multilayer nanoemulsions presents a significantly lower $(p<0.05)$ amount of FFA released when compared with the nanoemulsion, suggesting that the multilayer nanoemulsions were digested at a slower rate. At this stage, chitosan reduced the lipid digestion from $77.67 \pm 1.19 \%$ to $65.29 \pm 2.92 \%$, being these results in agreement with previous studies (Klinkesorn and McClements, 2009; Pinheiro et al., 2016; Tokle et al., 2012). The decrease of the FFA release at jejunum when using chitosan layer may be explained by the ability of bile salts to form electrostatic complex with chitosan, reducing the digestibility of the lipids by sterically hindering the lipase (Klinkesorn and Julian McClements, 2010; Klinkesorn and McClements, 2009). Also, large aggregates of chitosan could restrict the access of lipase to the lipids (McClements 


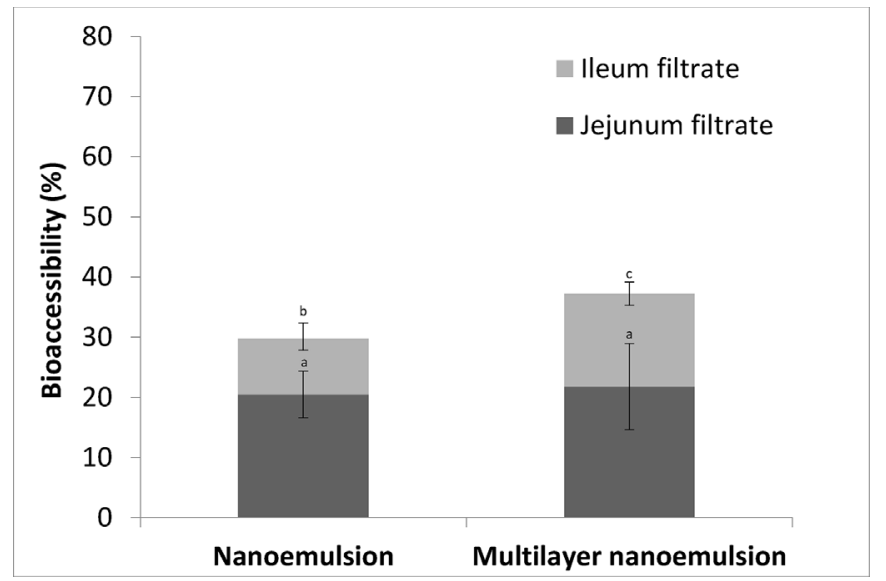

Fig. 6. Percentage of bioaccessibility as the nanosystems undergo through jejunal and ileal stages of the dynamic in vitro digestion. Each value represents mean $\pm \operatorname{SD}(n=3)$; One Way Analysis of Variance (ANOVA) coupled with the Tukey's post-hoc analysis to identify means with significant differences ( $p$ value of $p<0.05$ was considered significant); ${ }^{\text {a-c }}$ Different superscripts indicate significant differences among samples.

and Li, 2010; Zhang et al., 2015).

These results suggest that the presence of a chitosan layer on the nanoemulsions may be useful to control the rate of lipid digestion and FFA adsorption within the gastrointestinal tract, although being inefficient in preventing lipid digestion.

\subsubsection{Influence of chitosan layer on curcumin bioaccessibility}

Curcumin bioaccessibility from the nanosystems was measured based on the curcumin concentration present in mixed micelles after digestion at jejunum and ileal filtrate samples. Curcumin bioaccessibility increases during the digestion once the FFA, monoacyglycerides and diacylglycerides produced are able to form mixed micelles capable of solubilize curcumin (Pinheiro et al., 2016). Fig. 6 shows that the use of a chitosan layer significantly increased $(p<0.05)$ the curcumin bioaccessibility, reaching a value of $37.2 \pm 7.6 \%$ for multilayer nanoemulsions when compared to $29.8 \pm 0.5 \%$ for the nanoemulsions' bioaccessibility (Fig. 6). For both nanosystems, the bioaccessibility of curcumin was higher in the jejunal filtrate, when compared to the ileal filtrate fraction. When comparing the results of the curcumin bioaccessibility at ileal filtrate between nanoemulsions and multilayer nanoemulsions, it can be observed that multilayer nanoemulsions presented higher $(p<0.05)$ bioaccessibility of curcumin regarding to nanoemulsions (15.5 $\pm 1.92 \%$ and $9.3 \pm 2.63 \%$, respectively). These results are in agreement with the results of FFA released from lipid digestion, Fig. 5, i.e. the higher curcumin bioaccessibility observed in the ileal filtrate for the multilayer nanoemulsions can be related to the higher amount of FFA observed in the ileal filtrate. Since the access of lipases to lipids is crucial for a good bioaccessibility, the observed higher amount of FFA that could be part of mixed micelles could increase the solubility of curcumin, increasing therefore its bioaccessibility (Pinheiro et al., 2016; Porter et al., 2007). Free curcumin (i.e. curcumin solubilized in MCTs) bioaccessibility was also evaluated (data not shown), achieving only a value of $0.15 \pm 0.01 \%$ at the jejunal filtrate, while there was no evidence of curcumin at the ileal filtrate fraction. Therefore, both nanosystems were able to increase curcumin bioaccessibility.

\subsection{Cytotoxicity assay}

The cytotoxicity of curcumin, nanosystems and chitosan were assessed using MTS test through the evaluation of Caco-2 cells viability. As shown in Fig. 7, free curcumin and nanosystems were not toxic relatively to the control group (cells with RPMI medium), indicating that none of the samples had effect in Caco- 2 cell viability after $4 \mathrm{~h}$ of incubation in the tested concentrations (between 4.8 and 19.0 $\mu \mathrm{g}_{\text {curcumin }} \cdot \mathrm{mL}^{-1}$ ). Similar results showing that curcumin do not present any cytotoxicity have been obtained in a previous work (Silva et al., 2018).

Furthermore, chitosan did not present toxicity towards the control group (data not shown). These results ensure the safety of curcumin and nanosystems to be further tested for cellular antioxidant activity and for permeability studies.

\subsection{Cellular antioxidant activity}

Curcumin is a well-known antioxidant compound (Pinheiro et al., 2016; Siviero et al., 2015; Zou et al., 2015). Several in vitro chemical antioxidant assays have been performed to evaluate the antioxidant activity of curcumin in the presence of different free radicals (Ak and Gülçin, 2008; Jayaprakasha et al., 2006). Nonetheless, the wide usage of these antioxidant chemical assays do not account for some physiological conditions that happens in vivo, such as, cell uptake, metabolism and distribution of the bioactive compounds (Wolfe and Liu, 2007). For this reason, in this work the CAA of free curcumin and curcumin entrapped within nanosystems was quantified using the human cell model Caco-2. Table 1 presents the $E C_{50}\left(\mu \mathrm{g}_{\text {curcumin }} \cdot \mathrm{mL}^{-1}\right)$ and $C A A$

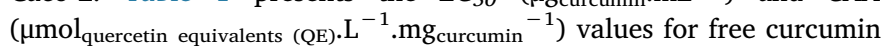
and curcumin entrapped in the nanosystems. It shows that curcumin encapsulated in the nanosystems exhibited a significantly $(p<0.05)$ higher CAA value than curcumin solubilized in ethanol $(0.21 \pm 0.01$ $\mu \mathrm{mol}_{\mathrm{QE}} \cdot \mathrm{L}^{-1} \cdot \mathrm{mg}_{\text {curcumin }}{ }^{-1}$ ). Nanosystems exhibited 8.5 and 9.9-fold higher values of CAA for nanoemulsions and multilayer nanoemulsions, $1.79 \pm 0.08$ and $2.08 \pm 0.16 \mu \mathrm{mol}_{\mathrm{QE}} \cdot \mathrm{L}^{-1} . \mathrm{mg}_{\text {curcumin }}{ }^{-1}$, respectively. Results suggest that nanoencapsulation of curcumin using WPI as emulsifier lead to an increase of the cellular antioxidant activity of curcumin in Caco- 2 cells. The use of a chitosan layer did not have significant impact $(p>0.05)$ in the $C A A$ value, suggesting that chitosan did not enhance the cellular antioxidant capacity of curcumin. The low $C A A$ promoted by the free curcumin may be explained by the fact that curcumin was solubilized in ethanol and cell media, which could have limited the solubility of curcumin. On the other hand, curcumin encapsulated in the nanosystems has a larger surface area, therefore increasing the CAA values (Sessa et al., 2011; Yu et al., 2011). Another explanation may be the fact that curcumin suffers rapid hydrolysis at weak basic conditions (ethanol and media) that may induce their rapid degradation. On the contrary, both nanosystems stabilized curcumin against hydrolysis (Yu et al., 2011). Other authors suggested that micelle encapsulation enhanced the CAA of curcuminoids in HepG2 cell line (liver hepatocellular carcinoma cells) by 2 -fold when compared to free curcuminoids solubilized in DMSO (Yu et al., 2011), which is in accordance with the present work.

\subsection{Permeability assay}

The transport mechanisms of curcumin loaded in the nanosystems were evaluated using Caco- 2 cell monolayers, aiming at simulating the small intestine epithelium. The selected concentration of curcumin for the permeability studies was $19 \mu \mathrm{g} \mathrm{mL}^{-1}$ since at this concentration the nanosystems did not show cytotoxicity. The $P_{\text {app }}$ of most compounds ranges between $1.0 \times 10^{-7}$ and $1.0 \times 10^{-5} \mathrm{~cm} \mathrm{~s}^{-1}$. Usually a poorly transported compound exhibits a $P_{a p p}$ value of $\sim 1.0 \times 10^{-7} \mathrm{~cm} \mathrm{~s}^{-1}$, whereas a well transported compound has a $P_{a p p}$ value of $\sim 1.0 \times 10^{-5} \mathrm{~cm} \mathrm{~s}^{-1}$. Otherwise, average permeable compounds will exhibit $P_{\text {app }}$ values of $\sim 1.0 \times 10^{-6} \mathrm{~cm} \mathrm{~s}^{-1}$ (Gao et al., 2001; Sessa et al., 2014). Curcumin permeation rates for nanoemulsions and multilayer nanoemulsions are showed in Fig. 8a). The permeation rate for curcumin nanoemulsions was determined as $1.25 \pm 0.05 \times 10^{-6} \mathrm{~cm} \mathrm{~s}^{-1}$, that falls in the range of the averagely 


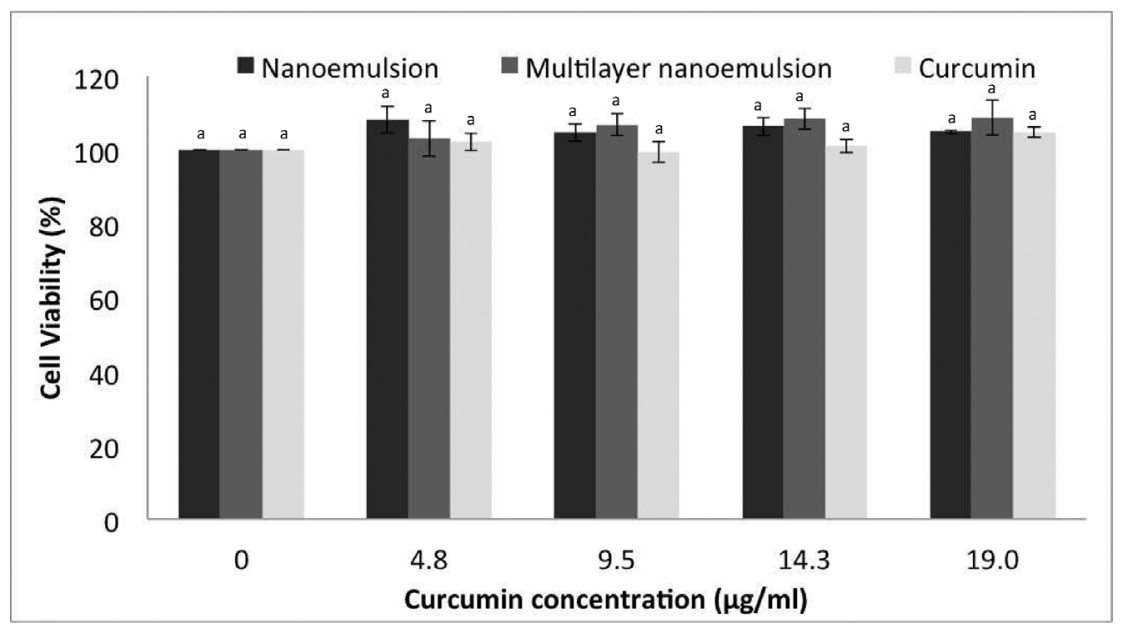

Fig. 7. Cell viability in percentage in function of curcumin concentration in terms of $\mu$ g. $\mathrm{mL}^{-1}$. Each value represents mean $\pm \mathrm{SD}(\mathrm{n}=3$ ); One Way Analysis of Variance (ANOVA) coupled with the Tukey's post-hoc analysis to identify means with significant differences ( $p$ value of $p<0.05$ was considered significant).

Table 1

$E C_{50}$ and CAA values for the inhibition of Peroxyl Radical-Induced DCFH Oxidation by curcumin and nanoemulsions.

\begin{tabular}{lll}
\hline Samples & $E C_{50}\left(\mu \mathrm{g}_{\text {curcumin }} \cdot \mathrm{mL}^{-1}\right)$ & $\begin{array}{l}\mathrm{CAA}\left(\mu \mathrm{mol}_{\mathrm{QE}} \cdot \mathrm{L}^{-1} \cdot \mathrm{mg}_{\text {curcumin }}\right. \\
\end{array}$ \\
\hline Curcumin & $16.4 \pm 0.6^{\mathrm{a}}$ & $0.21 \pm 0.01^{\mathrm{a}}$ \\
Nanoemulsion & $1.97 \pm 0.09^{\mathrm{b}}$ & $1.79 \pm 0.08^{\mathrm{b}}$ \\
Multilayer & $1.69 \pm 0.13^{\mathrm{b}}$ & $2.08 \pm 0.16^{\mathrm{b}}$ \\
$\quad$ & & \\
\hline
\end{tabular}

Each value represents mean $\pm \mathrm{SD}(\mathrm{n}=3)$; One Way Analysis of Variance (ANOVA) coupled with the Tukey's post-hoc analysis to identify means with significant differences ( $p$ value of $p<0.05$ was considered significant); ${ }^{\text {ab }}$ Different superscripts within the same column indicate significant differences among samples.

permeable compounds. The rate of permeation of curcumin across the cells monolayer increased as function of time (data not shown) and the TEER values (not shown) did not change significantly $(p>0.05)$, suggesting that curcumin permeated the cells monolayer through transcellular pathway (Li et al., 2015a; Sun et al., 2015; Yu and Huang, 2012). Nevertheless, these results do not show if nanoemulsions permeate directly across the Caco- 2 cell monolayers ( $\mathrm{Yu}$ and Huang, 2012). Also, the permeation rate for curcumin multilayer nanoemulsions was determined as $1.93 \pm 0.02 \times 10^{-6} \mathrm{~cm} \mathrm{~s}^{-1}$ (Figs. 8a), 1.55fold higher $(p<0.05)$ than the value obtained for nanoemulsions. Chitosan is known for its ability to facilitate the widening of tight junctions, enhancing the paracellular transport of bioactive compounds, such as curcumin, across the Caco- 2 cells monolayer (Smith et al., 2004; Ting et al., 2014). In our study, the presence of chitosan significantly decreased $(p<0.05)$ the TEERS value, suggesting that curcumin multilayer nanoemulsions were also able to directly diffuse through Caco- 2 cell tight junctions. A reduction of $\sim 90 \%$ in TEER values can indicate that nanosystems could disrupt the tight junctions, enhancing the transport of the bioactive compounds via paracellular pathway (Li et al., 2015b; Wang et al., 2015). Despite the reduction of the TEER values, Caco-2 cells monolayer did not show visual signs of damage. Thus, these results suggest that both transcellular and paracellular pathways may exist at the same time when nanoemulsions with a chitosan layer are absorbed.

In addition, the cellular uptake of curcumin delivered by nanoemulsions and multilayer nanoemulsions was quantified (Fig. 8b)). The use of a chitosan layer significantly increased the cellular uptake by endocytosis from $4.44 \pm 0.11 \%$ to $10.50 \pm 0.15 \%$, which represents an increase of 2.36-fold on the cellular uptake. Since size and surface

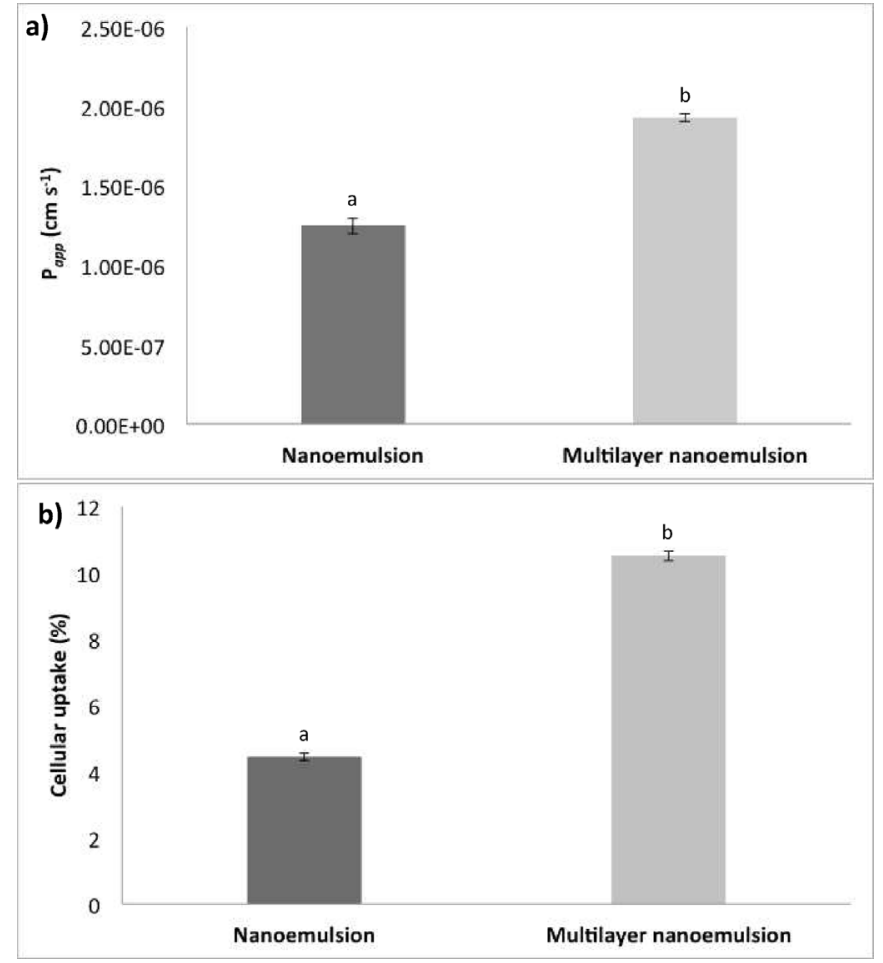

Fig. 8. a) Apparent permeability coefficient $\left(P_{a p p}\right)$ for nanoemulsions and multilayer nanoemulsions; and b) Cellular uptake in percentage for nanoemulsions and multilayer nanoemulsions. Each value represents mean \pm SD $(\mathrm{n}=3)$; One Way Analysis of Variance (ANOVA) coupled with the Tukey's posthoc analysis to identify means with significant differences ( $p$ value of $p<0.05$ was considered significant); ${ }^{a-b}$ Different superscripts indicate significant differences among samples.

properties (such as charge and hydrophobicity) of the nanosystems governs the cellular uptake, this result may be explained by the positive charges of multilayer nanoemulsions, due to chitosan amino groups, being the multilayer nanoemulsions internalized into the Caco- 2 cells, whereas the negative charge of WPI, due to electrostatic repulsions, can decrease the uptake efficiency (Li et al., 2015b). Hydrophilic polyelectrolytes such as chitosan can enhance nanosystems transport through Caco-2 cells via specific interaction between nanosystems and intestinal epithelium (des Rieux et al., 2006; McClements, 2015). For instance, other authors reported that negative nanoparticles, due to 
repulsions between the nanoparticles and negative charged HeLa cells, had a lower cellular uptake, when compared with positive charged nanoparticles (Harush-Frenkel et al., 2007; Li et al., 2015b).

The mass balance performed (data not shown) presented a low value of curcumin recovery (between $30 \%$ and $40 \%$ ), which suggests that curcumin could have been metabolized by the Caco-2 cells, accumulated in the cell monolayer, bind to the plate, as well as lost during the washing step or during sample preparation for HPLC (Li et al., 2015b; McClements, 2015). After being internalized in the Caco-2 cells, curcumin nanosystems may have been consumed in metabolic processes such as reduction, conjugation, dehydroxylation, cyclization, and methylation(Li et al., 2015b; McClements, 2015). In fact, curcumin undergoes metabolic O-conjugation to curcumin glucuronide and curcumin sulfate and bioreduction to tetrahydrocurcumin, hexahydrocurcumin, octahydrocurcumin, and hexahydrocurcuminol (Prasad et al., 2014).

\section{Conclusions}

The purpose of this study was to develop lipid-based nanosystems for curcumin encapsulation, while evaluating the effect of the addition of a chitosan layer on nanosystems stability during storage and in vitro digestion (i.e. lipids' digestibility and curcumin bioaccessibility). Also the cytotoxicity, cellular antioxidant activity and transport properties through a Caco- 2 cell monolayer of undigested nanosystems were evaluated. Results showed that both nanosystems (nanoemulsions and multilayer nanoemulsions) can increase the bioaccessibility of curcumin. Also, WPI-stabilized nanoemulsions and multilayer nanoemulsions can significantly increase the cellular antioxidant activity of curcumin, suggesting that both systems can be used for the delivery of water-insoluble curcumin. Permeability assays performed in Caco2 cells showed that the use of chitosan can enhance the apparent permeability coefficient of curcumin by 1.55 -fold. Although the permeation rate of multilayer nanoemulsions was higher than that of nanoemulsions, both the transcellular and paracellular pathways may exist at the same time.

This study suggests that food grade lipid-based nanosystems can be designed as delivery systems offering the opportunity to create functional foods able to maximize curcumin antioxidant capacity.

\section{Acknowledgements}

The authors Hélder D. Silva, Ana C. Pinheiro and Miguel A. Cerqueira (SFRH/BD/81288/2011, SFRH/BPD/101181/2014 and SFRH/BPD/72753/2010, respectively) are the recipients of a fellowship from the Foundation for Science and Technology. This work was supported by Portuguese Foundation for Science and Technology under the scope of the Project PTDC/AGR-TEC/5215/2014, of the strategic funding of UID/BIO/04469/2013 unit and COMPETE 2020 (POCI-010145-FEDER-006684), BioTecNorte operation (NORTE-01-0145FEDER-000004) funded by the European Regional Development Fund under the scope of Norte2020 - Programa Operacional Regional do Norte, PEst-OE/EQB/LA0004/2011 and iNOVA4Health - UID/Multi/ $04462 / 2013$, a program financially supported by FCT/Ministério da Educação e Ciência, through national funds and co-funded by FEDER under the PT2020 Partnership Agreement. The authors also acknowledge the European Commission: BIOCAPS (316265, FP7/REGPOT2012-2013.1). The support of EU Cost Action FA1001 is gratefully acknowledged. The authors also acknowledge Stepan (The Netherlands) for providing the Neobee 1053 oil, and Rui Fernandes from IBMC, University of Porto for assistance in taking the TEM pictures.

\section{References}

Acevedo-Fani, A., Soliva-Fortuny, R., Martín-Belloso, O., 2017. Nanostructured emulsions and nanolaminates for delivery of active ingredients: improving food safety and functionality. Trends Food Sci. Technol. 60, 12-22.

Adamczak, M., Kupiec, A., Jarek, E., Szczepanowicz, K., Warszyński, P., 2014. Preparation of the squalene-based capsules by membrane emulsification method and polyelectrolyte multilayer adsorption. Colloid. Surface. Physicochem. Eng. Aspect. 462, 147-152.

Ahmed, K., Li, Y., McClements, D.J., Xiao, H., 2012. Nanoemulsion- and emulsion-based delivery systems for curcumin: encapsulation and release properties. Food Chem. 132 (2), 799-807.

Ak, T., Gülçin, İ., 2008. Antioxidant and radical scavenging properties of curcumin. Chem. Biol. Interact. 174 (1), 27-37.

Aoki, T., Decker, E.A., McClements, D.J., 2005. Influence of environmental stresses on stability of $\mathrm{O} / \mathrm{W}$ emulsions containing droplets stabilized by multilayered membranes produced by a layer-by-layer electrostatic deposition technique. Food Hydrocolloids 19 (2), 209-220.

Artiga-Artigas, M., Lanjari-Pérez, Y., Martín-Belloso, O., 2018. Curcumin-loaded nanoemulsions stability as affected by the nature and concentration of surfactant. Food Chem. 266, 466-474.

Burke, S.E., Barrett, C.J., 2003a. Acid - Base equilibria of weak polyelectrolytes in multilayer thin films. Langmuir 19 (8), 3297-3303.

Burke, S.E., Barrett, C.J., 2003b. Ph-responsive properties of multilayered poly(l-lysine)/ hyaluronic acid surfaces. Biomacromolecules 4 (6), 1773-1783.

Cerqueira, M., Pinheiro, A., Silva, H., Ramos, P., Azevedo, M., Flores-López, M., Rivera, M., Bourbon, A., Ramos, Ó., Vicente, A., 2014. Design of bio-nanosystems for oral delivery of functional compounds. Food Engineering Reviews 6 (1-2), 1-19.

Cui, J., van Koeverden, M.P., Müllner, M., Kempe, K., Caruso, F., 2014. Emerging methods for the fabrication of polymer capsules. Adv. Colloid Interface Sci. 207, 14-31.

des Rieux, A., Fievez, V., Garinot, M., Schneider, Y.-J., Préat, V., 2006. Nanoparticles as potential oral delivery systems of proteins and vaccines: a mechanistic approach. J. Contr. Release 116 (1), 1-27.

Gao, J., Hugger, E.D., Beck-Westermeyer, M.S., Borchardt, R.T., 2001. Estimating Intestinal Mucosal Permeation of Compounds Using Caco-2 Cell Monolayers, Current Protocols in Pharmacology. John Wiley \& Sons, Inc.

Guzey, D., McClements, D.J., 2006. Formation, stability and properties of multilayer emulsions for application in the food industry. Adv. Colloid Interface Sci. 128-130, $227-248$.

Harush-Frenkel, O., Debotton, N., Benita, S., Altschuler, Y., 2007. Targeting of nanoparticles to the clathrin-mediated endocytic pathway. Biochem. Biophys. Res. Commun. 353 (1), 26-32.

Hu, M., Li, Y., Decker, E., Xiao, H., McClements, D., 2011. Impact of layer structure on physical stability and lipase digestibility of lipid droplets coated by biopolymer nanolaminated coatings. Food Biophys. 6 (1), 37-48.

Jayaprakasha, G.K., Jaganmohan Rao, L., Sakariah, K.K., 2006. Antioxidant activities of curcumin, demethoxycurcumin and bisdemethoxycurcumin. Food Chem. 98 (4), $720-724$.

Jelezova, I., Drakalska, E., Momekova, D., Shalimova, N., Momekov, G., Konstantinov, S., Rangelov, S., Pispas, S., 2015. Curcumin loaded pH-sensitive hybrid lipid/block copolymer nanosized drug delivery systems. Eur. J. Pharmaceut. Sci. 78, 67-78.

Kaur, K., Kumar, R., Mehta, S.K., 2015. Nanoemulsion: a new medium to study the interactions and stability of curcumin with bovine serum albumin. J. Mol. Liq. 209, 62-70.

Klinkesorn, U., Julian McClements, D., 2010. Impact of lipase, bile salts, and polysaccharides on properties and digestibility of tuna oil multilayer emulsions stabilized by lecithin-chitosan. Food Biophys. 5 (2), 73-81.

Klinkesorn, U., McClements, D.J., 2009. Influence of chitosan on stability and lipase digestibility of lecithin-stabilized tuna oil-in-water emulsions. Food Chem. 114 (4), 1308-1315.

Langerholc, T., Maragkoudakis, P.A., Wollgast, J., Gradisnik, L., Cencic, A., 2011. Novel and established intestinal cell line models - an indispensable tool in food science and nutrition. Trends Food Sci. Technol. 22 (1), S11-S20.

Li, M., Cui, J., Ngadi, M.O., Ma, Y., 2015a. Absorption mechanism of whey-protein-delivered curcumin using Caco-2 cell monolayers. Food Chem. 180, 48-54.

Li, Y., Hu, M., Du, Y., Xiao, H., McClements, D.J., 2011. Control of lipase digestibility of emulsified lipids by encapsulation within calcium alginate beads. Food Hydrocolloids 25 (1), 122-130.

Li, Y., Hu, M., Xiao, H., Du, Y., Decker, E.A., McClements, D.J., 2010. Controlling the functional performance of emulsion-based delivery systems using multi-component biopolymer coatings. Eur. J. Pharm. Biopharm. 76 (1), 38-47.

Li, Z., Jiang, H., Xu, C., Gu, L., 2015b. A review: using nanoparticles to enhance absorption and bioavailability of phenolic phytochemicals. Food Hydrocolloids 43, 153-164.

Liu, Y., Cai, Y., Jiang, X., Wu, J., Le, X., 2016. Molecular interactions, characterization and antimicrobial activity of curcumin-chitosan blend films. Food Hydrocolloids 52, 564-572.

Madrigal-Carballo, S., Lim, S., Rodriguez, G., Vila, A.O., Krueger, C.G., Gunasekaran, S., Reed, J.D., 2010. Biopolymer coating of soybean lecithin liposomes via layer-by-layer self-assembly as novel delivery system for ellagic acid. Journal of Functional Foods 2 (2), 99-106.

Maljaars, J., Peters, H.P.F., Haddeman, E., Masclee, A., 2009. M2078 distribution of small intestinal fat delivery influences satiety and food intake. Gastroenterology 136 (5) A480.

Malvern, I., 2011. Dynamic Light Scattering Common Terms Defined.

Matias, A., Nunes, S.L., Poejo, J., Mecha, E., Serra, A.T., Madeira, P.J.A., Bronze, M.R., Duarte, C.M.M., 2014. Antioxidant and anti-inflammatory activity of a flavonoid-rich concentrate recovered from Opuntia ficus-indica juice. Food \& Function 5 (12), $3269-3280$. 
McClements, D.J., 2013. Edible lipid nanoparticles: digestion, absorption, and potential toxicity. Prog. Lipid Res. 52 (4), 409-423.

McClements, D.J., 2015. Encapsulation, protection, and release of hydrophilic active components: potential and limitations of colloidal delivery systems. Adv. Colloid Interface Sci. 219, 27-53.

McClements, D.J., Li, Y., 2010. Structured emulsion-based delivery systems: controlling the digestion and release of lipophilic food components. Adv. Colloid Interface Sci. 159 (2), 213-228.

McClements, D.J., Xiao, H., 2012. Potential biological fate of ingested nanoemulsions: influence of particle characteristics. Food \& Function 3 (3), 202-220.

Minekus, M., Jelier, M., Xiao, J.-z., Kondo, S., Iwatsuki, K., Kokubo, S., Bos, M., Dunnewind, B., Havenaar, R., 2005. Effect of partially hydrolyzed guar gum (PHGG) on the bioaccessibility of fat and cholesterol. Biosc. Biotech. Biochem. 69 (5), 932-938.

Mohammed, M.A., Syeda, J.T.M., Wasan, K.M., Wasan, E.K., 2017. An overview of chitosan nanoparticles and its application in non-parenteral drug delivery. Pharmaceutics 9 (4), 53.

Mora-Huertas, C.E., Fessi, H., Elaissari, A., 2010. Polymer-based nanocapsules for drug delivery. Int. J. Pharm. 385 (1-2), 113-142.

Mu, H., Høy, C.-E., 2004. The digestion of dietary triacylglycerols. Prog. Lipid Res. 43 (2), 105-133.

Mun, S., Decker, E.A., McClements, D.J., 2005. Influence of droplet characteristics on the formation of oil-in-water emulsions stabilized by Surfactant - Chitosan layers. Langmuir 21 (14), 6228-6234.

Ozturk, B., Argin, S., Ozilgen, M., McClements, D.J., 2014. Formation and stabilization of nanoemulsion-based vitamin $\mathrm{E}$ delivery systems using natural surfactants: quillaja saponin and lecithin. J. Food Eng. 142 (0), 57-63.

Pantaleone, D., Yalpani, M., Scollar, M., 1992. Unusual susceptibility of chitosan to enzymic hydrolysis. Carbohydr. Res. 237, 325-332.

Pinheiro, A.C., Coimbra, M.A., Vicente, A.A., 2016. In vitro behaviour of curcumin nanoemulsions stabilized by biopolymer emulsifiers - effect of interfacial composition. Food Hydrocolloids 52, 460-467.

Pinsirodom, P., P, K.L., 2005. Lipase assays. In: Wrolstad, R.E. (Ed.), Handbook of Food Analytical Chemistry. Wiley, Hoboken, NJ, pp. 371-383.

Plaza-Oliver, M., Baranda, J.F.S.d., Rodríguez Robledo, V., Castro-Vázquez, L., GonzalezFuentes, J., Marcos, P., Lozano, M.V., Santander-Ortega, M.J., Arroyo-Jimenez, M.M., 2015. Design of the interface of edible nanoemulsions to modulate the bioaccessibility of neuroprotective antioxidants. Int. J. Pharm. 490 (1-2), 209-218.

Porter, C.J.H., Trevaskis, N.L., Charman, W.N., 2007. Lipids and lipid-based formulations: optimizing the oral delivery of lipophilic drugs. Nat. Rev. Drug Discov. 6 (3), 231-248.

Prasad, S., Tyagi, A.K., Aggarwal, B.B., 2014. Recent developments in delivery, bioavailability, absorption and metabolism of curcumin: the golden pigment from golden spice. Cancer Res Treat 46 (1), 2-18.

Raj, S., Shankaran, D.R., 2016. Curcumin based biocompatible nanofibers for lead ion detection. Sensor. Actuator. B Chem. 226, 318-325.

Rodrigues, R.M., Martins, A.J., Ramos, O.L., Malcata, F.X., Teixeira, J.A., Vicente, A.A., Pereira, R.N., 2015. Influence of moderate electric fields on gelation of whey protein isolate. Food Hydrocolloids 43, 329-339.

Salvia-Trujillo, L., Sun, Q., Um, B.H., Park, Y., McClements, D.J., 2015. In vitro and in vivo study of fucoxanthin bioavailability from nanoemulsion-based delivery systems: impact of lipid Carrier type. Journal of Functional Foods 17, 293-304.

Serra, A.T., 2010. Valorization of Traditional Portuguese Apples and Cherries Biochemical Characterization and Development of Functional Ingredients. Instituto de Tecnologia Química e Biológica. Universidade Nova de Lisboa.

Serra, A.T., Duarte, R.O., Bronze, M.R., Duarte, C.M.M., 2011a. Identification of bioactive response in traditional cherries from Portugal. Food Chem. 125 (2), 318-325.

Serra, A.T., Matias, A.A., Almeida, A.P.C., Bronze, M.R., Alves, P.M., de Sousa, H.C., Duarte, C.M.M., 2011b. Processing cherries (Prunus avium) using supercritical fluid technology. Part 2. Evaluation of SCF extracts as promising natural chemotherapeutical agents. J. Supercrit. Fluids 55 (3), 1007-1013.

Serra, A.T., Poejo, J., Matias, A.A., Bronze, M.R., Duarte, C.M.M., 2013. Evaluation of Opuntia spp. derived products as antiproliferative agents in human colon cancer cell line (HT29). Food Res. Int. 54 (1), 892-901.

Sessa, M., Balestrieri, M.L., Ferrari, G., Servillo, L., Castaldo, D., D'Onofrio, N., Donsì, F.,
Tsao, R., 2014. Bioavailability of encapsulated resveratrol into nanoemulsion-based delivery systems. Food Chem. 147 (0), 42-50.

Sessa, M., Tsao, R., Liu, R., Ferrari, G., Donsì, F., 2011. Evaluation of the stability and antioxidant activity of nanoencapsulated resveratrol during in vitro digestion. J. Agric. Food Chem. 59 (23), 12352-12360.

Shimizu, M., 2010. Interaction between food substances and the intestinal epithelium. Biosc. Biotech. Biochem. 74 (2), 232-241.

Shin, S.S., Lee, Y.C., Lee, C., 2001. The degradation of chitosan with the aid of lipase from Rhizopus Japonicus for the production of soluble chitosan. J. Food Biochem. 25 (4), 307-321.

Silva, H.D., Cerqueira, M.A., Vicente, A.A., 2015. Influence of surfactant and processing conditions in the stability of oil-in-water nanoemulsions. J. Food Eng. 167, 89-98.

Silva, H.D., Poejo, J., Pinheiro, A.C., Donsì, F., Serra, A.T., Duarte, C.M.M., Ferrari, G., Cerqueira, M.A., Vicente, A.A., 2018. Evaluating the behaviour of curcumin nanoemulsions and multilayer nanoemulsions during dynamic in vitro digestion. Journal of Functional Foods 48, 605-613.

Siviero, A., Gallo, E., Maggini, V., Gori, L., Mugelli, A., Firenzuoli, F., Vannacci, A., 2015. Curcumin, a golden spice with a low bioavailability. J. Herb. Med. 5 (2), 57-70.

Smith, J., Wood, E., Dornish, M., 2004. Effect of chitosan on epithelial cell tight junctions. Pharmaceut. Res. 21 (1), 43-49.

Sun, Y., Xia, Z., Zheng, J., Qiu, P., Zhang, L., McClements, D.J., Xiao, H., 2015. Nanoemulsion-based delivery systems for nutraceuticals: influence of Carrier oil type on bioavailability of pterostilbene. Journal of Functional Foods 13, 61-70.

Szczepanowicz, K., Bazylińska, U., Pietkiewicz, J., Szyk-Warszyńska, L., Wilk, K.A., Warszyński, P., 2015. Biocompatible long-sustained release oil-core polyelectrolyte nanocarriers: from controlling physical state and stability to biological impact. Adv. Colloid Interface Sci. 222, 678-691.

Ting, Y., Jiang, Y., Ho, C.-T., Huang, Q., 2014. Common delivery systems for enhancing in vivo bioavailability and biological efficacy of nutraceuticals. Journal of Functional Foods 7, 112-128.

Tokle, T., Lesmes, U., Decker, E.A., McClements, D.J., 2012. Impact of dietary fiber coatings on behavior of protein-stabilized lipid droplets under simulated gastrointestinal conditions. Food \& Function 3 (1), 58-66.

Vachoud, L., Zydowicz, N., Domard, A., 2000. Physicochemical behaviour of chitin gels Carbohydr. Res. 326 (4), 295-304.

Wang, H., Joseph, J.A., 1999. Quantifying cellular oxidative stress by dichlorofluorescein assay using microplate reader1. Free Radic. Biol. Med. 27 (5-6), 612-616.

Wang, J., Ma, W., Tu, P., 2015. The mechanism of self-assembled mixed micelles in improving curcumin oral absorption: in vitro and in vivo. Colloids Surfaces B Biointerfaces 133, 108-119.

Wolfe, K.L., Liu, R.H., 2007. Cellular antioxidant activity (CAA) assay for assessing antioxidants, foods, and dietary supplements. J. Agric. Food Chem. 55 (22), 8896-8907.

Yang, D., Wang, X.-Y., Ji, C.-M., Lee, K.-T., Shin, J.-A., Lee, E.-S., Hong, S.-T., 2014. Influence of Ginkgo biloba extracts and of their flavonoid glycosides fraction on the in vitro digestibility of emulsion systems. Food Hydrocolloids 42 (1), 196-203.

Yu, H., Huang, Q., 2012. Improving the oral bioavailability of curcumin using novel organogel-based nanoemulsions. J. Agric. Food Chem. 60 (21), 5373-5379.

Yu, H., Li, J., Shi, K., Huang, Q., 2011. Structure of modified E-polylysine micelles and their application in improving cellular antioxidant activity of curcuminoids. Food \& Function 2 (7), 373-380.

Zeng, Z., Shen, Z.L., Zhai, S., Xu, J.L., Liang, H., Shen, Q., Li, Q.Y., 2017. Transport of curcumin derivatives in Caco-2 cell monolayers. Eur. J. Pharm. Biopharm. 117, $123-131$.

Zhang, C., Xu, W., Jin, W., Shah, B.R., Li, Y., Li, B., 2015. Influence of anionic alginate and cationic chitosan on physicochemical stability and carotenoids bioaccessibility of soy protein isolate-stabilized emulsions. Food Res. Int. 77, 419-425.

Zhao, L., Du, J., Duan, Y., Zang, Y.n., Zhang, H., Yang, C., Cao, F., Zhai, G., 2012. Curcumin loaded mixed micelles composed of Pluronic P123 and F68: preparation, optimization and in vitro characterization. Colloids Surfaces B Biointerfaces 97 101-108.

Zou, L., Zheng, B., Liu, W., Liu, C., Xiao, H., McClements, D.J., 2015. Enhancing nutraceutical bioavailability using excipient emulsions: influence of lipid droplet size on solubility and bioaccessibility of powdered curcumin. Journal of Functional Foods 15, 72-83. 